\title{
CXCL12, A Potential Biomarker and A Vital Modulator of Tumor Immune Microenvironment (TIME) of Bladder Cancer: From A Comprehensive Analysis of TCGA Database
}

\section{Jinyan Wang}

The Affiliated Jiangning Hospital of Nanjing Medical University

Jinqiu Wang

Dafeng People's Hospital

\section{Quan Gu}

Nanjing Medical University

\section{Yan Yang}

The Affiliated Jiangning Hospital of Nanjing Medical University

\section{Yajun Ma}

The Affiliated Jiangning Hospital of Nanjing Medical University

quanan zhang ( $\square$ quananzhang_doctor@163.com)

The affiliated Jiangning hospital of Nanjing Medical university https://orcid.org/0000-0002-6356-7122

\section{Primary research}

Keywords: Bladder cancer, tumor immune microenvironment (TIME), tumor-infiltrating immune cells (TICs), CXCL12, biomarker

Posted Date: October 7th, 2020

DOI: https://doi.org/10.21203/rs.3.rs-84428/v1

License: (c) (i) This work is licensed under a Creative Commons Attribution 4.0 International License. Read Full License 


\section{Abstract}

Background: Tumor immune microenvironment (TIME) played a significant role in the initiation and progression of bladder cancer (BC). However, there are few researches regarding the association between immune-related genes (IRGs) and tumor-infiltrating immune cells (TICs) in TME of BC.

Methods: We calculated the proportion of immune/stromal component and TICs in TME of 414 BC samples and 19 normal samples downloaded from The Cancer Genome Atlas (TCGA) database with the help of ESTIMATE and CIBERSORT algorithms. Differentially expressed genes (DEGs) were obtained from the comparison between Stromal and Immune Score and further analyzed by GO and KEGG enrichment analysis, as well as protein-protein interaction (PPI) network and COX regression analysis. CXC chemokine ligand-12 (CXCL12) was overlapping from the above analysis. Afterwards, single gene analysis of CXCL12 was carried out through difference analysis, paired analysis and Gene Set Enrichment Analysis (GSEA). The association between the expression of CXCL12 and TICs was assessed by difference analysis and correlation analysis.

Results: The results indicated that immune and stromal component in TME of BC were associated with patients' clinic-pathological characteristics. We further confirmed that 284 DEGs were primarily enriched in immune-associated activities and CXCL12 was the most significant gene, which shared the leading nodes in PPI network and closely related with BC patients' survival. Single gene analysis revealed that CXCL12 was down-regulated in BC samples and significantly related with the clinic-pathological characteristics of patients. Further analysis indicated that CXCL12 greatly participated in immuneassociated activities through closely communicating with TICs in TIME of BC.

Conclusions: CXCL12 might be a potential biomarker and a vital modulator of TIME through communicating with multiple TICs, which might provide an extra insight for the immunotherapy of BC.

\section{Introduction}

Over the last 30 years, few advances have been made in the treatment of bladder cancer (BC) since the application of cisplatin-based chemotherapy in the mid-1980s ${ }^{[1]}$. Fortunately, immunotherapy has emerged as a novel potential therapy recently. Several clinical trials, such as IMvigor 210 study ${ }^{[2]}$ and CheckMate 275 study ${ }^{[3]}$, have revealed that $\mathrm{BC}$ patients greatly benefited from the treatment of immunecheckpoint blockade (ICB). Although multiple biomarkers have been associated with the prediction of immunotherapy effect, such as the expression of PD-L1 and TMB, it is still less than satisfactory to select $\mathrm{BC}$ patients who are likely to benefit most from immunotherapy ${ }^{[4]}$.

In recent years, the tumor microenvironment (TME) has caught our attention as its role in modulating the initiation and progression of cancers, including $\mathrm{BC}{ }^{[5-10]}$. TME is composed of nonmalignant cells, vessels, lymphoid organs or lymph nodes, nerves, intercellular components and metabolites ${ }^{[11]}$. In brief, stromal component and immune component constitute the TME ${ }^{[12]}$. Furthermore, accumulating 
researches indicated that tumor immune microenvironment (TIME) had great potential to influence tumor initiation, predict immunotherapeutic responsiveness and reveal new therapeutic targets ${ }^{[13]}$. Up to date, numerous studies revealed that immune-related genes (IRGs), obtained from TME, functioned to predict

the survival of cancer patients, including breast cancer ${ }^{[14]}$, endometrial cancer ${ }^{[15]}$, liver cancer ${ }^{[16]}$, gastric cancer ${ }^{[5]}$, bladder cancer ${ }^{[17]}$ and so on. For example, Q. Ding, et al. ${ }^{[18]}$ found that a nine-gene signature was closely related with immune infiltration in TME and the survival of patients with ovarian cancer. Besides, tumor-infiltrating immune cells (TICs) in TIME, such as tumor-infiltrating lymphocytes (TILs), tumor-associated macrophages (TAMs) and so on, have the potential to be biomarkers and predict the prognosis of multiple cancers ${ }^{[19-21]}$. For instance, elevated level of TILs were associated with better overall survival (OS), higher pathological complete response ( $(\mathrm{CR})$ rate and fewer recurrence, as well as greatly benefited from trastuzumab treatment in breast cancer ${ }^{[22-25]}$. Deficient CD4 $+T$ cell help suppressed the response of cytotoxic T lymphocytes (CTLs), which were able to establish efficient and durable antitumor activity ${ }^{[26]}$. TAMs acted to inhibit T cell recruitment and function as well as modulate the immunity of various tumors, thus affecting the response of immunotherapy ${ }^{[27]}$. Galectin-9 + TAMs predicted prognosis and response to adjuvant chemotherapy in BC patients ${ }^{[28]}$. However, there are few researches regarding the association between IRGs and TICs in the TME of BC. The exploration of the relationship between IRGs and TICs in TIME might provide us a new sight into the progression and immunotherapy of BC.

Fortunately, with the rapid development of transcriptome profiling based on functional genomics analysis, comprehensive analysis of IRGs and TICs in the TIME of BC has become possible. In our study, we applied ESTIMATE and CIBERSORT algorithms to calculate the ratio of immune and stromal component, as well as TICs proportion of BC samples from The Cancer Genome Atlas (TCGA) database. Next, we started with differentially expressed genes (DEGs) acquired by the comparison of immune and stromal component in BC samples, and found out that CXC chemokine ligand-12 (CXCL12) acted to be a potential biomarker of $\mathrm{BC}$ and a vital regulator of immune-associated activities in TIME of $\mathrm{BC}$ via communicating with multiple TICs.

\section{Methods}

\section{Data acquisition}

The transcriptome profiling and clinical data of $414 \mathrm{BC}$ samples and 19 normal samples were downloaded from TCGA database (https://portal.gdc.cancer.gov/).

\section{The assessment of immune component, stromal component and the total component in the TME of BC}

We applied ESTIMATE algorithm to calculate the proportion of immune and stromal component of each tumor sample in $\mathrm{R}$ language (version 3.6.3). Immune Score represented the ratio of immune component in TME of tumor samples. Stromal Score represented the ratio of stromal component in TME of tumor samples. ESTIMATE Score represented the sum of immune and stromal component in TME of tumor 
samples. The higher Score indicated the larger proportion of the corresponding immune or stromal component in TME.

\section{Difference analysis between Scores and the clinic-pathological characteristics}

Difference analysis was conducted to learn the correlation between Immune/Stromal/ESTIMATE Score and clinic-pathological characteristics, such as age, gender, grade, stage and TNM classification. It was also carried out to find the association between the expression of CXCL12 and clinic-pathological characteristics as well as the association between the expression of CXCL12 and TICs. Wilcoxon rank sum and Kruskal-Wallis rank sum test were applied for the comparison. $\mathrm{P}<0.05$ was considered to be statistically significant.

\section{DEGs acquisition}

414 BC samples were grouped into to two subgroups, including high Immune/Stromal Score group and low Immune/Stromal Score group based on the comparison with the median. Package limma in $\mathrm{R}$ was applied for the analysis. A fold change (FC, log2 (high-score /low-score) $)>2$ and false discovery rate (FDR) $<0.05$ were used to search the DEGs. Pheatmap package in $\mathrm{R}$ was used to plot the heatmaps of DEGs.

\section{Intersection analysis}

VennDiagram package in $\mathrm{R}$ was used to plot the venn diagram of DEGs.

\section{Gene ontology (GO) and Kyoto Encyclopedia of Genes and Genomes (KEGG) enrichment analysis}

GO and KEGG enrichment analysis of the above DEGs were further carried out by using the ClusterProfiler, enrichplot, and ggplot2 packages in $\mathrm{R}$. $\mathrm{P}<0.05$ was considered to be statistically significant.

\section{Protein-Protein Interaction (PPI) Network and Gene Set Enrichment Analysis (GSEA)}

The preliminary PPI network of 284 DEGs was acquired from the Search Tool for Retrieval of Interacting Genes/Proteins (STRING) database (version 11.0) and further reconstructed in Cytoscape (3.6.1). The confidence of interactive relationship of the nodes was $>0.95$. GSEA (4.1.0) based on the different gene sets, was applied to learn the specific functional profile of CXCL12. $P<0.05$ was considered to be statistically significant.

\section{CoX Regression Analysis}

Package survival in $\mathrm{R}$ was applied to conduct univariate COX regression.

\section{Difference analysis and paired analysis of CXCL12}

Package Beeswarm in R was used to assess the expression of CXCL12 in bladder tumor samples and normal samples. Packages Ggpubr and BiocManager were applied to learn the expression of CXCL12 in 
bladder tumor samples and the paired normal samples. Wilcoxon test was used for the comparison. $\mathrm{P}<$ 0.05 was considered to be statistically significant.

\section{Survival analysis}

Packages survival and survminer in R were used to carry out the survival analysis. Kaplan-Meier plot and log-rank tests were conducted to learn the associations between the expression of CXCL12 and the survival of $B C$ patients. $P<0.05$ was considered to be statistically significant.

\section{TICs Profile}

TICs profile in BC samples was evaluated by CIBERSORT algorithm.

\section{Results}

\section{Analysis process of the study}

We first downloaded the transcriptome profiling and clinical data of $414 \mathrm{BC}$ samples and 19 normal samples from TCGA database and further analyzed the ratio of immune and stromal component in TME of each tumor sample through ESTIMATE algorithm. Difference analysis was conducted to find out the association between immune/stromal component in TME and the clinic-pathological characteristics of $\mathrm{BC}$ patients. A total of $284 \mathrm{DEGs}$ were further acquired based on the immune and stromal component in TME of BC. GO and KEGG enrichment analysis of these 284 DEGs were performed to learn their biological functions. Finally, CXCL12 was found out through the intersection analysis of PPI network and univariate COX regression. Then, we focused on the expression profile of CXCL12 in BC samples and normal samples, and the association between the expression of CXCL12 and survival and clinic-pathological characteristics of BC patients. GSEA of different gene collections was also carried out to learn the functional profiles of CXCL12. TICs profile in TIME of BC samples was calculated by CIBERSORT algorithm. Difference analysis and correlation analysis were applied to find out the correlation between the expression of CXCL12 and TICs.

\section{Immune and stromal component in TME were associated with the clinic-pathological characteristics of $\mathrm{BC}$}

To learn the association between the proportion of immune/stromal component in TME and the clinicpathological characteristics of BC patients, we first set up the immune/stromal component evaluating system of TME in BC samples. Immune, Stromal and ESTIMATE Score represented the proportion of immune, stromal and the total component in TME of each tumor sample, respectively. The higher Score suggested the larger proportion of immune or stromal component in TME. The clinic-pathological characteristics of BC patients, including age, gender, grade, stage and TNM classification, were concluded in Supplement Table 1. Difference analysis revealed that Stromal Score was associated with patients' age and gender, especially the grade (Figure $2 a-c, p=0.018 ; 0.032 ;<0.001$ ). The higher Stromal Score indicated the higher grade of BC (Figure 2c, $p<0.001$ ). It was also found that Stromal Score was related 
with T, N classification and clinical stage of BC (Figure 2d-g). Regarding Immune Score, it was significantly up-regulated in female patients (Figure $2 \mathrm{i}, \mathrm{p}=0.037$ ) and the higher Immune Score also suggested the higher grade of $B C$ (Figure 2j, $\mathrm{p}<0.001$ ). However, neither TNM classification nor clinical stage of BC was associated with Immune Score (Figure 2k-n, p>0.05). As for ESTIMATE Score, the results similarly showed that it was up-regulated in female patients (Figure $2 p, p=0.028$ ) and indicated the higher grade of BC (Figure $2 q, p<0.001$ ). In addition, ESTIMATE Score was connected with T classification and clinical stage of $\mathrm{BC}$ (Figure 2r-u). From above, we might conclude that the stromal and immune component in TME were significantly associated with the grade of $\mathrm{BC}$, and partly related with TNM classification and clinical stage. This provided us a new sight to explore the underlying mechanisms of the development and progression of BC.

\section{DEGs were primarily enriched in immune-associated activities}

In order to further understand the underlying mechanisms of BC TME, we conducted difference analysis to acquire the DEGs profile in TME. BC samples were first divided into two groups, including high Stromal Score group and low Stromal Score group (or high Immune Score group and low Immune Score group). Heat map displayed the DEGs profile in TME of BC (Figure 3a-b). To be specific, there were 537 DEGs obtained from the comparison between high Stromal Score and low Stromal Score, among which 496 DEGs were up-regulated and 41 DEGs were down-regulated (Figure 3c, Supplement Table 2). Besides, there were 531 DEGs obtained from the comparison between high Immune Score and low Immune Score, among which 466 DEGs were up-regulated and 65 DEGs were down-regulated (Figure 3c, Supplement Table 3). In conclusion, a total of 284 DEGs were commonly up-regulated or down-regulated in Stromal or Immune Score group (Figure 3c, Supplement Table 4). Next, we carried out GO and KEGG enrichment analysis to assess the biological functions of these 284 DEGs. Go enrichment analysis revealed that 284 DEGs were primarily enriched in immune-associated activities, such as leukocyte proliferation, migration and chemotaxis, lymphocyte and mononuclear cell proliferation and so on (Figure 3d-f). Similarly, KEGG enrichment analysis suggested that these DEGs were mainly enriched in immune-associated activities, including complement and coagulation cascades, cytokine-cytokine receptor interaction, B cell receptor signaling pathway, chemokine signaling pathway and so on. Therefore, we considered that DEGs acquired from Stromal and Immune Score group of BC were significantly associated with immuneassociated activities in TME.

\section{PPI network and univariate COX regression analysis of 284 DEGs}

To learn the detailed reciprocity among these 284 DEGs, we further ploted PPI network through STRING database and cytoscape software. The potential interactions among DEGs were displayed in Figure 4a. The top ten DEGs, which shared the leading nodes, were ranked in Figure 4b, including ITGAM, CXCL12, CXCL13 and so on (Supplement Table 5). Univariate COX regression of DEGs was conducted at the same time to find out the specific DEGs, which were significantly associated with BC patients' survival (Figure 4c). MMP9, COMP, F13A1 and CXCL12 came to our eyes (Figure 4c, $p<0.05$; Supplement Table 6). Intersection analysis was finally carried out to search the DEGs who shared the leading nodes in PPI 
network and were significantly related with BC patients' survival (Figure 4d). Fortunately, CXCL12 emerged.

\section{CXCL12 was down-regulated in BC tissues and associated with the clinic-pathological characteristics}

The CXC chemokine CXCL12 greatly participated in multiple physiological and pathological processes through interacting with its receptors $\mathrm{CXC}$ chemokine receptor 4 (CXCR4) and atypical chemokine receptor 3 (ACKR3) ${ }^{[29]}$. In our study, CXCL12 was significantly down-regulated in BC tissues compared with normal tissues (Figure $5 a, p<0.001$ ). Besides, the paired analysis also indicated that CXCL12 was obviously down-regulated in $B C$ tissues compared with the paired normal tissues (Figure $5 b, p<0.001$ ). However, survival analysis suggested that the expression of CXCL12 was not significantly associated with the survival of $B C$ patients (Figure $5 c, p=0.050$ ). As for clinic-pathological characteristics of $B C$ patients, the results showed that the expression of CXCL12 changed with ages and higher expression of CXCL12 indicated the higher grade of BC (Figure $5 d, f ; p<0.001, p<0.001$ ). In addition, the expression of CXCL12 was partly correlated with T, N classification and clinical stages of BC (Figure 5h-k). Hence, we might conclude that $\mathrm{CXCL} 12$ had the potential to participate in the progression of $\mathrm{BC}$.

\section{CXCL12 greatly participated in immune-associated activities}

On account of the potential role of CXCL12 in the progression of $\mathrm{BC}$, we further explored the underlying mechanisms of CXCL12. BC cases were first divided into two groups, including high CXCL12 expression group and low CXCL12 expression group. GSEA suggested that for C5 collection, the gene ontology sets, genes in high CXCL12 expression group were enriched in cytokine binding, leukocyte migration and negative regulation of interleukin 6 production (Figure $6 a, p<0.05$ ). For $\mathrm{C} 2$ collection, the KEGG gene sets database, genes in high CXCL12 expression group were enriched in allograft rejection, antigen processing and presentation, B cell receptor signaling pathway, complement and coagulation cascades, and so on (Figure $6 b, p<0.05$ ). For hallmark gene sets, genes in high CXCL12 expression group were primarily enriched in allograft rejection, complement, IL2 stats signaling, IL6 jak stat3 signaling and so on. Finally, for $\mathrm{C7}$ collection defined by MSigDB, the immunologic gene sets, genes were found to be enriched in multiple immune-associated activities, which were related with CD4 T cell, CD8 T cell, B cell and so on (Figure $6 \mathrm{~d}, \mathrm{p}<0.05$ ). As a result, according to GSEA, CXCL12 was significantly related with immuneassociated activities and might play an important role in regulating TIME of BC.

\section{CXCL12 communicated with TICs in TIME}

Since GSEA suggested that CXCL12 was significantly correlated with immune-associated activities, we speculated that there might be underlying connections between CXCL12 and TIME of BC. In consequence, we calculated the proportion of TICs in BC samples through CIBERSORT algorithm. The specific ratio of 22 TICs in tumor samples was shown in Figure 7a. And the association among these TICs was exhibited in Figure 7b. Difference analysis was carried out to learn the association between the expression of CXCL12 and specific TICs. The results showed that 10 kinds of TICs, such as Macrophages M2, B cells naïve, T cells gamma delta, T cells CD4 naïve, T cells follicular helper and so on, were significantly 
associated with the expression of CXCL12 (Figure 7c, Supplement Table 7). The correlation analysis suggested that the expression of CXCL12 were positively or negatively related with 8 kinds of TICs, among which $B$ cells naïve, macrophages $\mathrm{M} 2$ and mast cells resting were positively associated, and dendritic cells activated, dendritic cells resting, NK cells resting, T cells CD4 naïve and T cells follicular helper were negatively associated (Figure 7e-I, p $<0.05$; Supplement Table 7). Finally, intersection analysis between difference analysis and correlation analysis suggested that 6 kinds of TICs were significantly related with the expression of CXCL12 (Figure 7d). In conclusion, CXCL12 was obviously related with various TICs in TIME of $B C$ and might greatly participated in regulating the TIME of BC via closely communicating with multiple TICs.

\section{Discussion}

In this study, we focused on the association setween IRGs and TICs in the TME of BC. Firstly, we downloaded the transcriptome profiling and clinical data of $414 \mathrm{BC}$ samples and 19 normal samples from TCGA database, and calculated the proportion of immune and stromal component in TME of each BC samples with the help of ESTIMATE algorithm. Secondly, difference analysis found out that Immune/Stromal Score was associated with the clinic-pathological characteristics of BC. Thirdly, DEGs were obtained through the comparison between high Immune Score and low Immune Score (or high Stromal Score and low Stromal Score), and GO and KEGG enrichment analysis suggested that these DEGs were mainly enriched in immune-related activities. Fourthly, PPI network and univariate COX regression analysis indicated that CXCL12 shared the leading nodes in PPI network and was significantly related with BC patients' survival. Fifthly, signal gene analysis was conducted and found out that CXCL12 was down-regulated in $\mathrm{BC}$ samples compared with normal sample and significantly related with the clinic-pathological characteristics of BC. GSEA revealed that CXCL12 was significantly associated with immune-associated activities and might play an important role in regulating TIME of BC. Finally, CIBERSORT algorithm was applied to calculate the proportion of TICs in BC samples, and difference analysis as well as correlation analysis suggested that CXCL12 was obviously connected with multiple TICs in TME of BC and might modulate the TIME of BC via closely communicating with TICs.

CXCL12 was traditionally classified as a homeostatic CXC chemokine and took a great part in modulating kinds of physiological and pathological processes via binding to its receptors CXCR4 and ACKR3 ${ }^{[29,30]}$. CXCL12/CXCR4 axis had been proved to be associated with the progression and therapy of cancers. For example, CXCL12/CXCR4 axis advanced pancreatic cancer development, invasion, and metastasis through complex crosstalk with other pathways, and was correlated with the poor prognosis of patients ${ }^{[31]}$. Treatment targeting the CXCL12/CXCR4 pathway increased the efficacy of radiotherapy of locally advanced cervical cancer ${ }^{[32]}$. The role of CXCL12 in tumor development mainly depended on the specific microenvironment of tumors ${ }^{[33]}$. In addition, it was found that the CXCL12-CXCR4/CXCR7 axis had a great influence in gastrointestinal malignancies through immune resistance ${ }^{[34]}$. Further researches suggested that the mechanisms of immunotherapy resistance might be associated with the CXCL12/CXCR4 axis ${ }^{[35]}$. Up to date, only few documents explored the association between CXCL12 and 
the tumorigenesis and progression of $\mathrm{BC}{ }^{[36]}$. Researches indicated that $\mathrm{CXCL} 12$ was down-regulated in $\mathrm{BC}$ tissues compared with normal bladder mucosal tissues, and positively associated with the differentiation degree and invasive depth of BC tissues ${ }^{[37]}$. Additionally, CXCL12/CXCR4 advanced invasion of $\mathrm{BC}$ cells through activating Stat3 ${ }^{[38]}$.

Furthermore, increasing studies have revealed that tumor-infiltrating immune cells played an important role in the progression and treatment of BC. For example, intratumoral TIGIT + CD8 + T-cell abundance functioned as a potential prognostic factor for patients' survival and a predictive biomarker for adjuvant chemotherapeutic effect ${ }^{[39]}$. CD19 + tumor-infiltrating B-cells activated CD4 + tumor-infiltrating T-cells in the TMB of $B C$ and acted as an independent prognostic factor for post-surgery survival and adjuvant chemotherapy benefits of BC patients ${ }^{[40]}$. Besides, DC-SIGN + TAM infiltration was significantly associated with a tumor-promoting TIME and might functioned as a prognostic indicator and therapeutic target in the immunotherapy of $\mathrm{BC}{ }^{[41]}$. However, there are no researches focusing on the associations between CXCL12 and TICs in TIME of BC.

In our study, we confirmed that CXCL12 was significantly down-regulated in BC tissues and associated with patients' clinic-pathological characteristics. In addition, functional analysis revealed that CXCL12 greatly participated in immune-associated activities and might regulate TIME of BC through communicating with multiple TICs, such as macrophages M2, B cells naïve, T cells follicular helper, mast cells resting, dendritic cells resting and T cells CD4 naïve. Further researches should be carried out to clarify the accuracy of the above combined analysis, and focused on the underlying mechanisms of the communication between CXCL12 and TICs in TIME of BC.

\section{Declarations}

\section{Ethics approval and consent to participate}

Not applicable.

\section{Consent for publication}

Not applicable.

\section{Availability of data and material}

The datasets generated and analyzed in this research are available in TCGA database (https://portal.gdc.cancer.gov)

\section{Competing interests}

The authors declare that they have no competing interests.

\section{Fundings}


This research was funded by the Natural Science Foundation of Jiangsu Province, grant number BK20161110, the Priority Academic Program Development of Jiangsu Higher Education Institutions, grant number JX10231802, Postgraduate Research and Practice Innovation Program of Jiangsu Province, grant number SJCX17_0387, the Science Foundation of Jiangsu Health vocational college, grant number JKC201948, the Science and Technology Development Fund of Nanjing Medical University, grant number NMUB2019235, the Nanjing health science and Technology Development Fund, grant number YKK18201, Science and technology development fund of Nanjing Medical University, grant number 2017NJMUZD091 and the Research and development fund of Kangda College of Nanjing Medical University, grant number KD2017KYJJYB017.

Author Contributions $₫$ Conceptualization, J.W.(Jinyan Wang) and Q.Z.; methodology, J.W.(Jinqiu Wang); software, Q.G.; validation, Q.Z.; formal analysis, J.W.(Jinyan Wang); investigation, J.W.(Jinqiu Wang).; resources, Q.G.; data curation, Q.G..; writing-original draft preparation, J.W.(Jinyan Wang), J.W.(Jinqiu Wang) and Q.Z.; writing-review and editing, J.W.(Jinyan Wang) and Q.Z.; supervision, Y.Y.; project administration, Y.M; funding acquisition, J.W.(Jinyan Wang), Y.Y. and Q.Z. All authors have read and agreed to the published version of the manuscript.

\section{Acknowledgment}

Not applicable.

\section{References}

1. Pardo JC, Ruiz de Porras V, Plaja A, Carrato C, Etxaniz O, Buisan O, et al. Moving towards Personalized Medicine in Muscle-Invasive Bladder Cancer: Where Are We Now and Where Are We Going? Int J Mol Sci. 2020; 21.

2. Rosenberg JE, Hoffman-Censits J, Powles T, van der Heijden MS, Balar AV, Necchi A, et al. Atezolizumab in patients with locally advanced and metastatic urothelial carcinoma who have progressed following treatment with platinum-based chemotherapy: a single-arm, multicentre, phase 2 trial. Lancet. 2016;387:1909-20.

3. Sharma P, Retz M, Siefker-Radtke A, Baron A, Necchi A, Bedke J, et al. Nivolumab in metastatic urothelial carcinoma after platinum therapy (CheckMate 275): a multicentre, single-arm, phase 2 trial. Lancet Oncol. 2017;18:312-22.

4. Hussain SA, Birtle A, Crabb S, Huddart R, Small D, Summerhayes M, et al. From Clinical Trials to Reallife Clinical Practice: The Role of Immunotherapy with PD-1/PD-L1 Inhibitors in Advanced Urothelial Carcinoma. Eur Urol Oncol. 2018;1:486-500.

5. Cai WY, Dong ZN, Fu XT, Lin LY, Wang L, Ye GD, et al. Identification of a Tumor Microenvironmentrelevant Gene set-based Prognostic Signature and Related Therapy Targets in Gastric Cancer. Theranostics. 2020;10:8633-47. 
6. Joshi S. Targeting the Tumor Microenvironment in Neuroblastoma: Recent Advances and Future Directions. Cancers (Basel). 2020; 12.

7. Wang $S$, Li Y, Xing C, Ding C, Zhang H, Chen $L$, et al. Tumor microenvironment in chemoresistance, metastasis and immunotherapy of pancreatic cancer. Am J Cancer Res. 2020;10:1937-53.

8. Yang $Y$, Yang Y, Yang J, Zhao X, Wei X. Tumor Microenvironment in Ovarian Cancer: Function and Therapeutic Strategy. Front Cell Dev Biol. 2020;8:758.

9. Zhang Z, Karthaus WR, Lee YS, Gao VR, Wu C, Russo JW, et al. Tumor Microenvironment-Derived NRG1 Promotes Antiandrogen Resistance in Prostate Cancer. Cancer Cell. 2020; 38: 279 - 96.e9.

10. Lin T, Zhang Q, Yuan A, Wang B, Zhang F, Ding Y, et al. Synergy of Tumor Microenvironment Remodeling and Autophagy Inhibition to Sensitize Radiation for Bladder Cancer Treatment. Theranostics. 2020;10:7683-96.

11. Jin MZ, Jin WL. The updated landscape of tumor microenvironment and drug repurposing. 2020; 5: 166.

12. Zhang L, Chen J, Cheng T, Yang H, Li H, Pan C. Identification of the key genes and characterizations of Tumor Immune Microenvironment in Lung Adenocarcinoma (LUAD) and Lung Squamous Cell Carcinoma (LUSC). J Cancer. 2020;11:4965-79.

13. Binnewies $\mathrm{M}$, Roberts EW, Kersten $\mathrm{K}$, Chan V. Understanding the tumor immune microenvironment (TIME) for effective therapy. 2018; 24: 541-50.

14. Zhao Y, Pu C, Liu Z. Exploration the Significance of a Novel Immune-Related Gene Signature in Prognosis and Immune Microenvironment of Breast Cancer. Front Oncol. 2020;10:1211.

15. Wang G, Wang D, Sun M, Liu X, Yang Q. Identification of prognostic and immune-related gene signatures in the tumor microenvironment of endometrial cancer. Int Immunopharmacol. 2020;88:106931.

16. Liu X, Niu X, Qiu ZA, Five-Gene. Signature Based on Stromal/Immune Scores in the Tumor Microenvironment and Its Clinical Implications for Liver Cancer. DNA Cell Biol. 2020;39:1621-38.

17. Luo Y, Zeng G, Wu S. Identification of Microenvironment-Related Prognostic Genes in Bladder Cancer Based on Gene Expression Profile. Front Genet. 2019;10:1187.

18. Ding Q, Dong S, Wang R, Zhang K, Wang $H$, Zhou $X$, et al. A nine-gene signature related to tumor microenvironment predicts overall survival with ovarian cancer. Aging. 2020;12:4879-95.

19. Bense RD, Sotiriou C, Piccart-Gebhart MJ, Haanen J, van Vugt M, de Vries EGE, et al. Relevance of Tumor-Infiltrating Immune Cell Composition and Functionality for Disease Outcome in Breast Cancer. J Natl Cancer Inst. 2017; 109.

20. Nam SJ, Kim YH, Park JE, Ra YS, Khang SK, Cho YH, et al. Tumor-infiltrating immune cell subpopulations and programmed death ligand 1 (PD-L1) expression associated with clinicopathological and prognostic parameters in ependymoma. Cancer Immunol Immunother. 2019;68:305-18. 
21. Liu X, Wu S, Yang Y, Zhao M, Zhu G, Hou Z. The prognostic landscape of tumor-infiltrating immune cell and immunomodulators in lung cancer. Biomed Pharmacother. 2017;95:55-61.

22. Loi S, Michiels S, Salgado R, Sirtaine N, Jose V, Fumagalli D, et al. Tumor infiltrating lymphocytes are prognostic in triple negative breast cancer and predictive for trastuzumab benefit in early breast cancer: results from the FinHER trial. Ann Oncol. 2014;25:1544-50.

23. Salgado R, Denkert C, Campbell C, Savas P, Nuciforo P, Aura C, et al. Tumor-Infiltrating Lymphocytes and Associations With Pathological Complete Response and Event-Free Survival in HER2-Positive Early-Stage Breast Cancer Treated With Lapatinib and Trastuzumab: A Secondary Analysis of the NeoALTTO Trial. JAMA Oncol. 2015;1:448-54.

24. Denkert C, Loibl S, Noske A, Roller M, Müller BM, Komor M, et al. Tumor-associated lymphocytes as an independent predictor of response to neoadjuvant chemotherapy in breast cancer. $\mathrm{J}$ Clin Oncol. 2010;28:105-13.

25. Denkert C, von Minckwitz G, Brase JC, Sinn BV, Gade S, Kronenwett R, et al. Tumor-infiltrating lymphocytes and response to neoadjuvant chemotherapy with or without carboplatin in human epidermal growth factor receptor 2-positive and triple-negative primary breast cancers. J Clin Oncol. 2015;33:983-91.

26. Borst J, Ahrends T, Bąbała N, Melief CJM, Kastenmüller W. CD4(+) T cell help in cancer immunology and immunotherapy. Nat Rev Immunol. 2018;18:635-47.

27. DeNardo DG, Ruffell B. Macrophages as regulators of tumour immunity and immunotherapy. Nat Rev Immunol. 2019;19:369-82.

28. Qi Y, Chang Y, Wang Z, Chen L, Kong Y, Zhang P, et al. Tumor-associated macrophages expressing galectin-9 identify immunoevasive subtype muscle-invasive bladder cancer with poor prognosis but favorable adjuvant chemotherapeutic response. 2019; 68: 2067-80.

29. Janssens R, Struyf S, Proost P. The unique structural and functional features of CXCL12. Cell Mol Immunol. 2018;15:299-311.

30. Janssens R, Struyf S, Proost P. Pathological roles of the homeostatic chemokine CXCL12. Cytokine Growth Factor Rev. 2018;44:51-68.

31. Sleightholm RL, Neilsen BK, Li J, Steele MM, Singh RK, Hollingsworth MA, et al. Emerging roles of the CXCL12/CXCR4 axis in pancreatic cancer progression and therapy. Pharmacol Ther. 2017;179:15870.

32. Lecavalier-Barsoum M, Chaudary N, Han K, Koritzinsky M, Hill R, Milosevic M. Targeting the CXCL12/CXCR4 pathway and myeloid cells to improve radiation treatment of locally advanced cervical cancer. Int J Cancer. 2018;143:1017-28.

33. Mortezaee K. CXCL12/CXCR4 axis in the microenvironment of solid tumors: A critical mediator of metastasis. Life Sci. 2020;249:117534.

34. Daniel SK, Seo YD, Pillarisetty VG. The CXCL12-CXCR4/CXCR7 axis as a mechanism of immune resistance in gastrointestinal malignancies. Semin Cancer Biol. 2020;65:176-88. 
35. Zhou W, Guo S, Liu M, Burow ME, Wang G. Targeting CXCL12/CXCR4 Axis in Tumor Immunotherapy. Curr Med Chem. 2019;26:3026-41.

36. Nazari A, Khorramdelazad H, Hassanshahi G. Biological/pathological functions of the CXCL12/CXCR4/CXCR7 axes in the pathogenesis of bladder cancer. Int J Clin Oncol. 2017;22:9911000 .

37. Yang DL, Xin MM, Wang JS, Xu HY, Huo Q, Tang ZR, et al. Chemokine receptor CXCR4 and its ligand CXCL12 expressions and clinical significance in bladder cancer. Genet Mol Res. 2015;14:17699-707.

38. Shen HB, Gu ZQ, Jian K, Qi J. CXCR4-mediated Stat3 activation is essential for CXCL12-induced cell invasion in bladder cancer. Tumour Biol. 2013;34:1839-45.

39. Liu Z, Zhou Q, Wang Z, Zhang H, Zeng H, Huang Q, et al. Intratumoral TIGIT(+) CD8(+) T-cell infiltration determines poor prognosis and immune evasion in patients with muscle-invasive bladder cancer. 2020; 8.

40. Jiang Q, Fu Q, Chang Y, Liu Z, Zhang J, Xu L, et al. CD19(+) tumor-infiltrating B-cells prime CD4(+) Tcell immunity and predict platinum-based chemotherapy efficacy in muscle-invasive bladder cancer. 2019; 68: 45-56.

41. Hu B, Wang Z. Blockade of DC-SIGN(+) Tumor-Associated Macrophages Reactivates Antitumor Immunity and Improves Immunotherapy in Muscle-Invasive Bladder Cancer. 2020; 80: 1707-19.

\section{Figures}




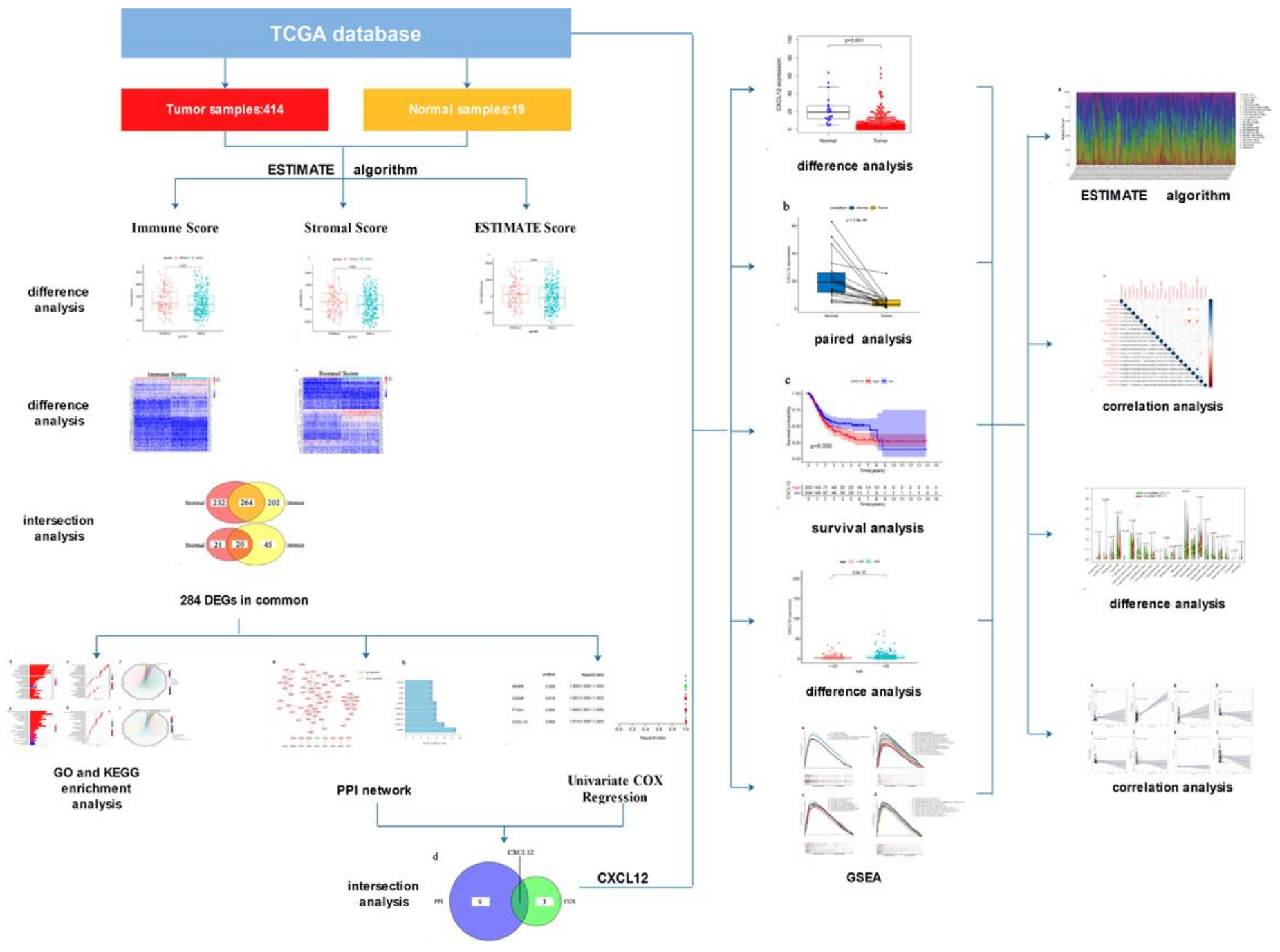

Figure 1

The analysis process of the study. 


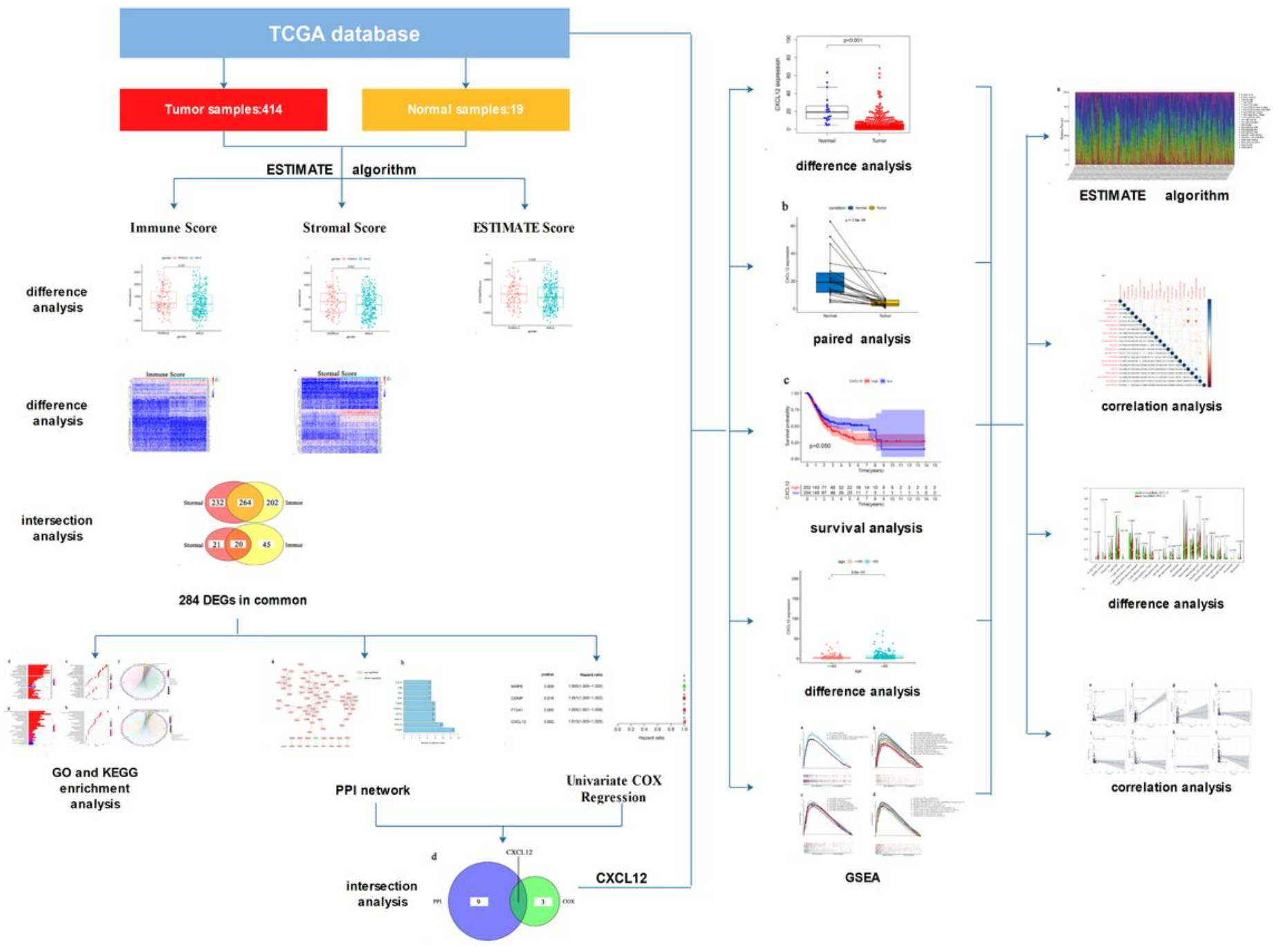

Figure 1

The analysis process of the study. 


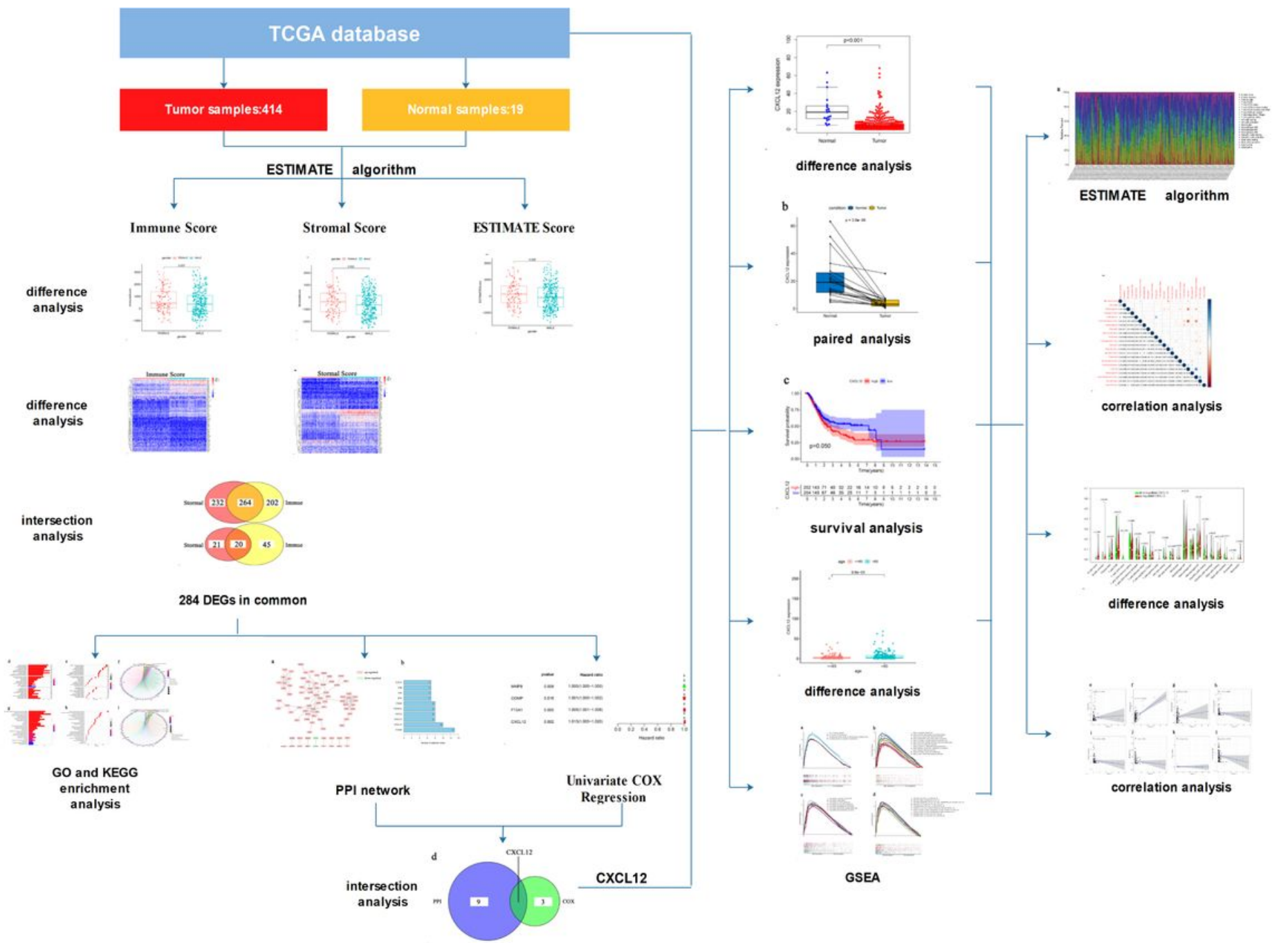

Figure 1

The analysis process of the study. 

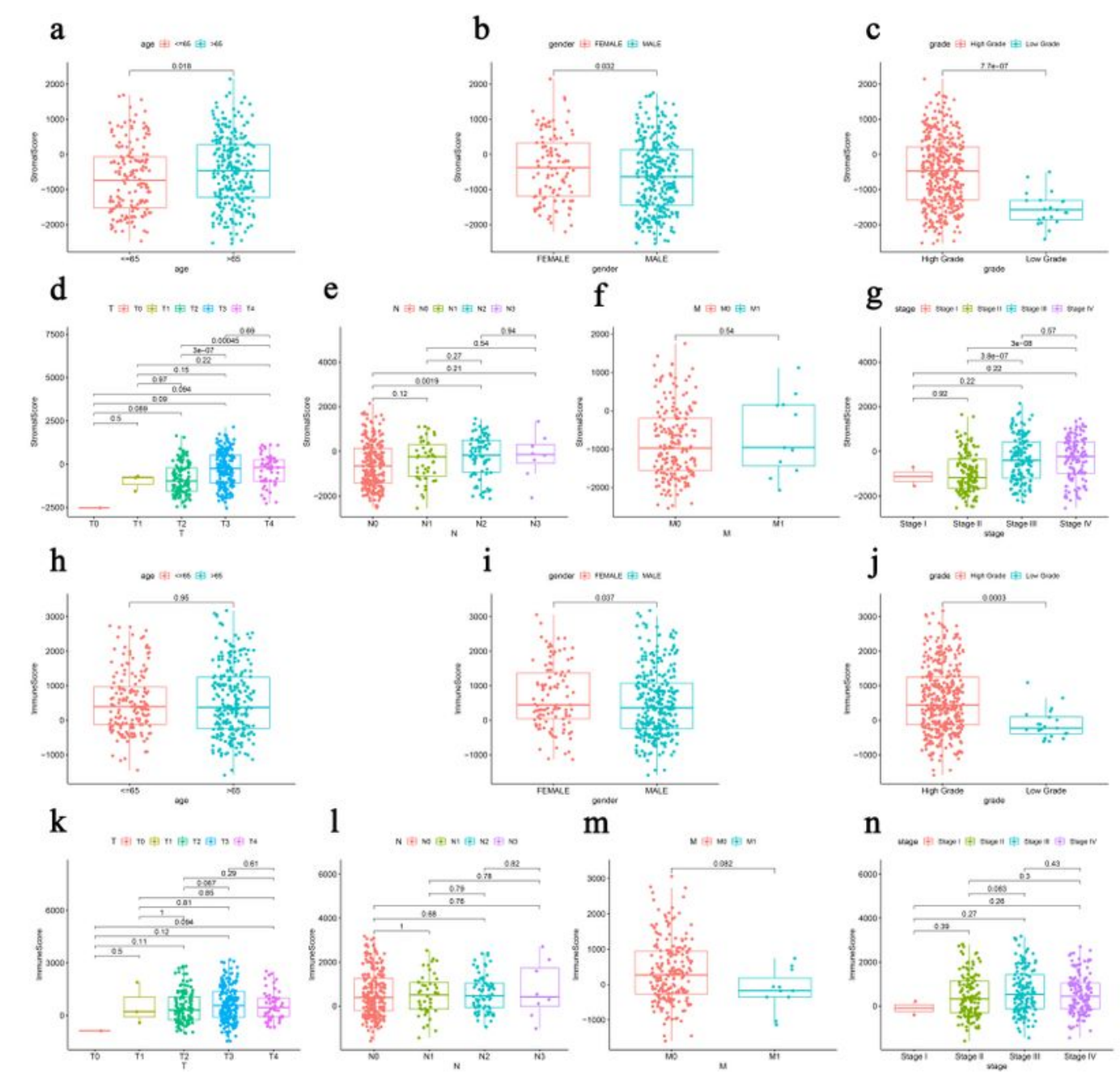

$\mathrm{m}$
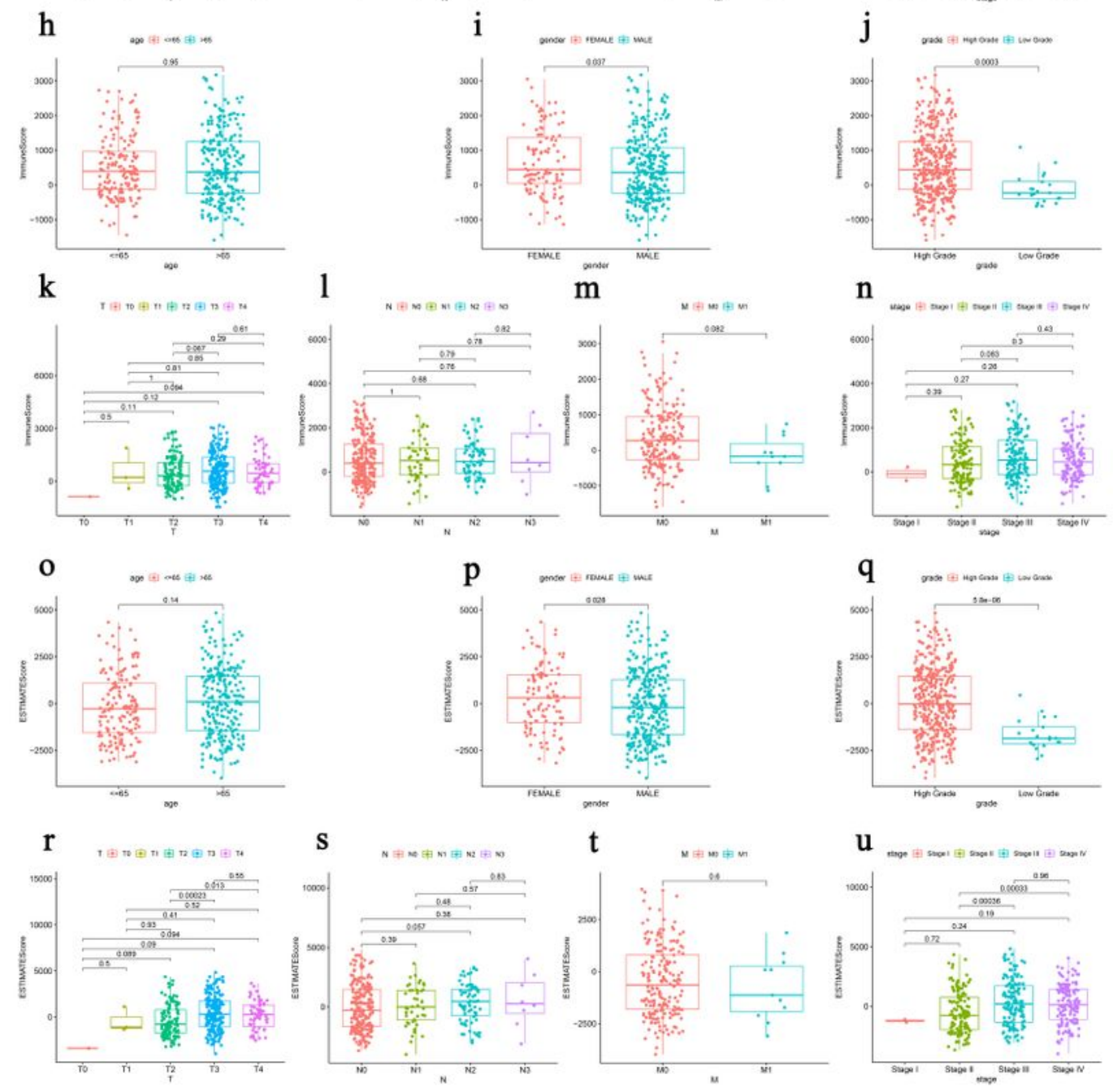

\section{Figure 2}

The relationship between Scores and clinic-pathological characteristics of BC patients. (a-g) The relationship between Stromal Score and clinic-pathological characteristics of BC patients, including age, gender, grade, TNM classification and stage; (h-n) The relationship between Immune Score and clinicpathological characteristics of BC patients; (o-u) The relationship between ESTIMATE Score and clinicpathological characteristics of BC patients. P-value was showed in each plot. 

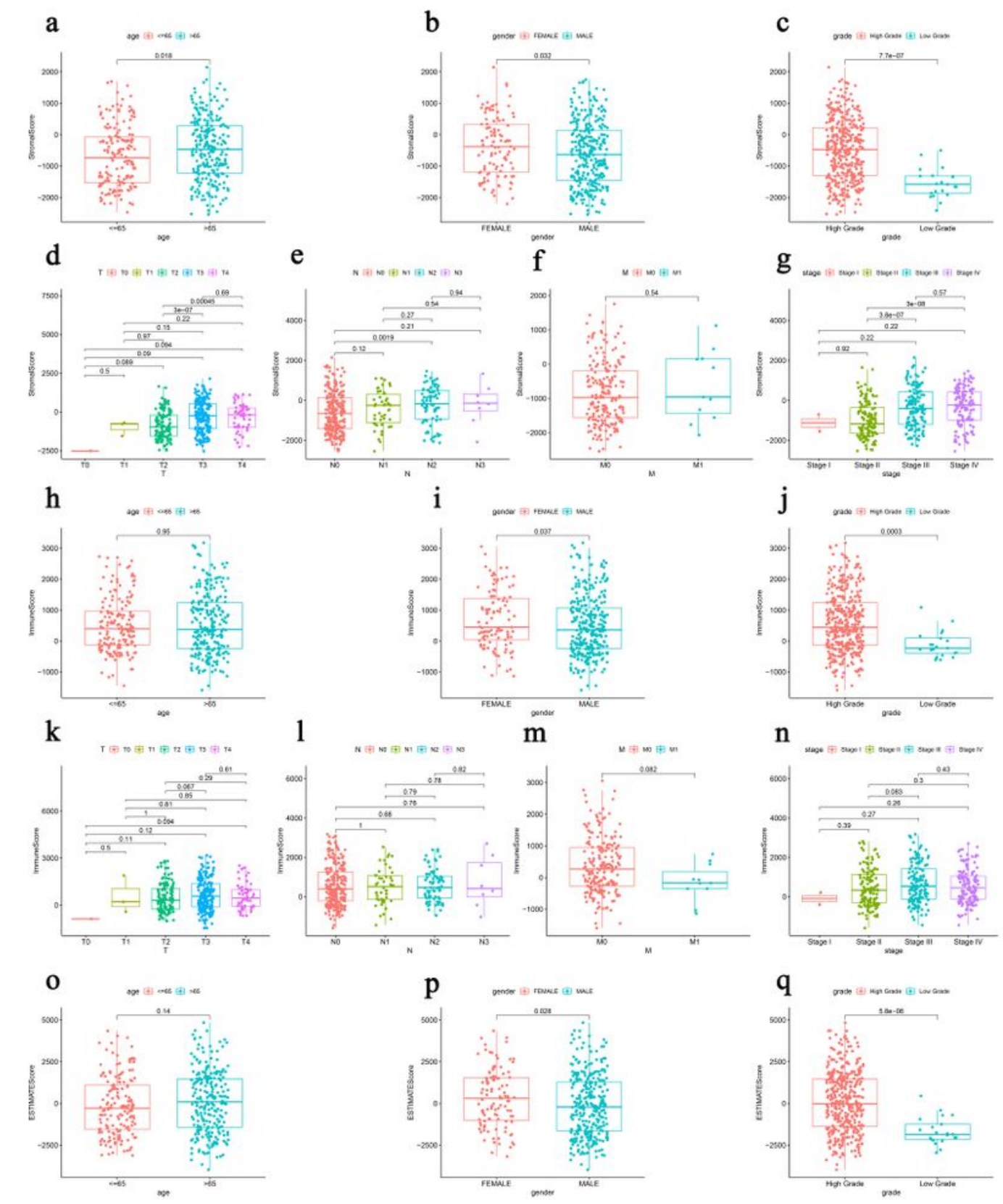

$\mathrm{m}$
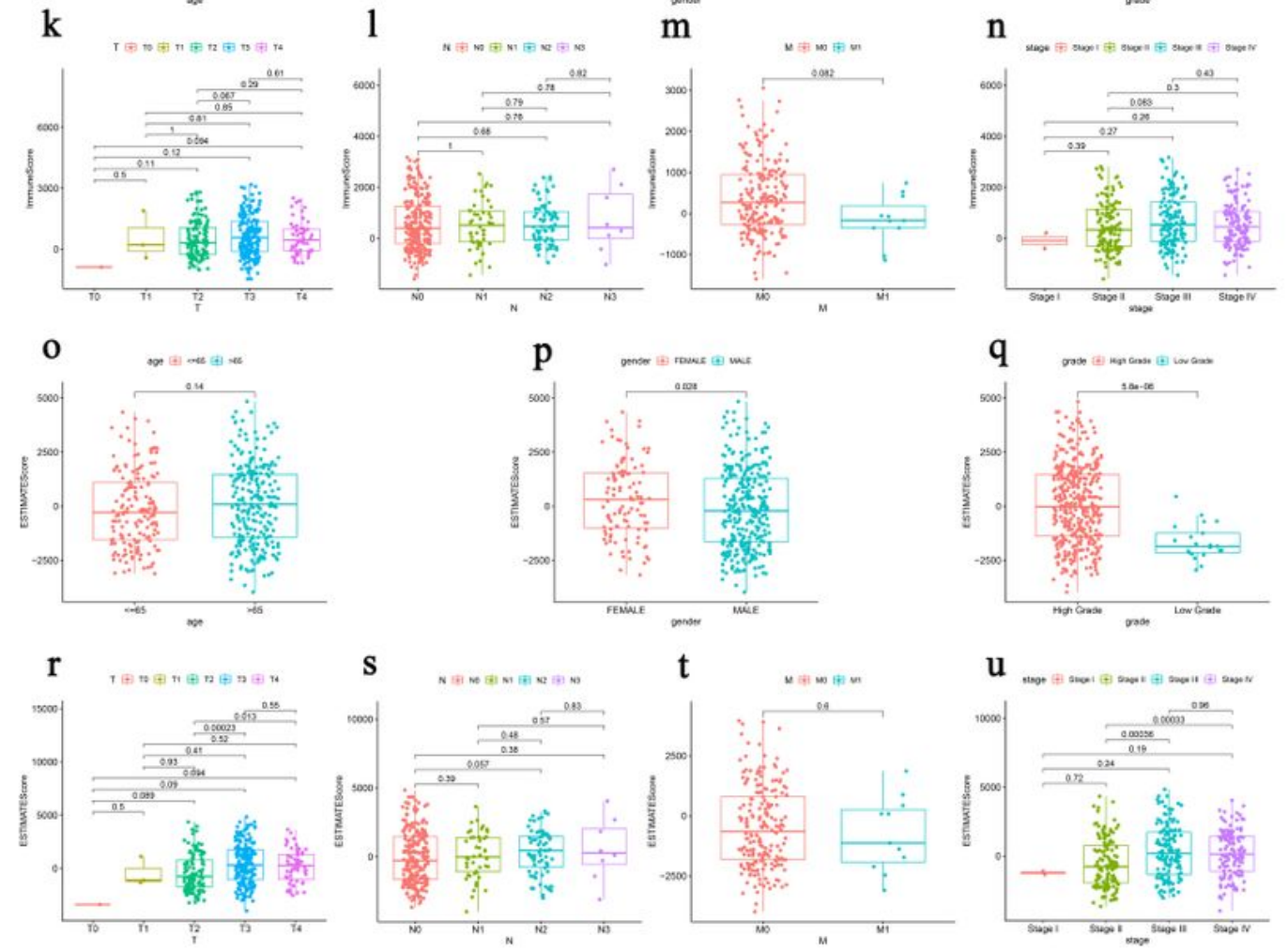

\section{Figure 2}

The relationship between Scores and clinic-pathological characteristics of BC patients. (a-g) The relationship between Stromal Score and clinic-pathological characteristics of BC patients, including age, gender, grade, TNM classification and stage; (h-n) The relationship between Immune Score and clinicpathological characteristics of BC patients; (o-u) The relationship between ESTIMATE Score and clinicpathological characteristics of BC patients. P-value was showed in each plot. 

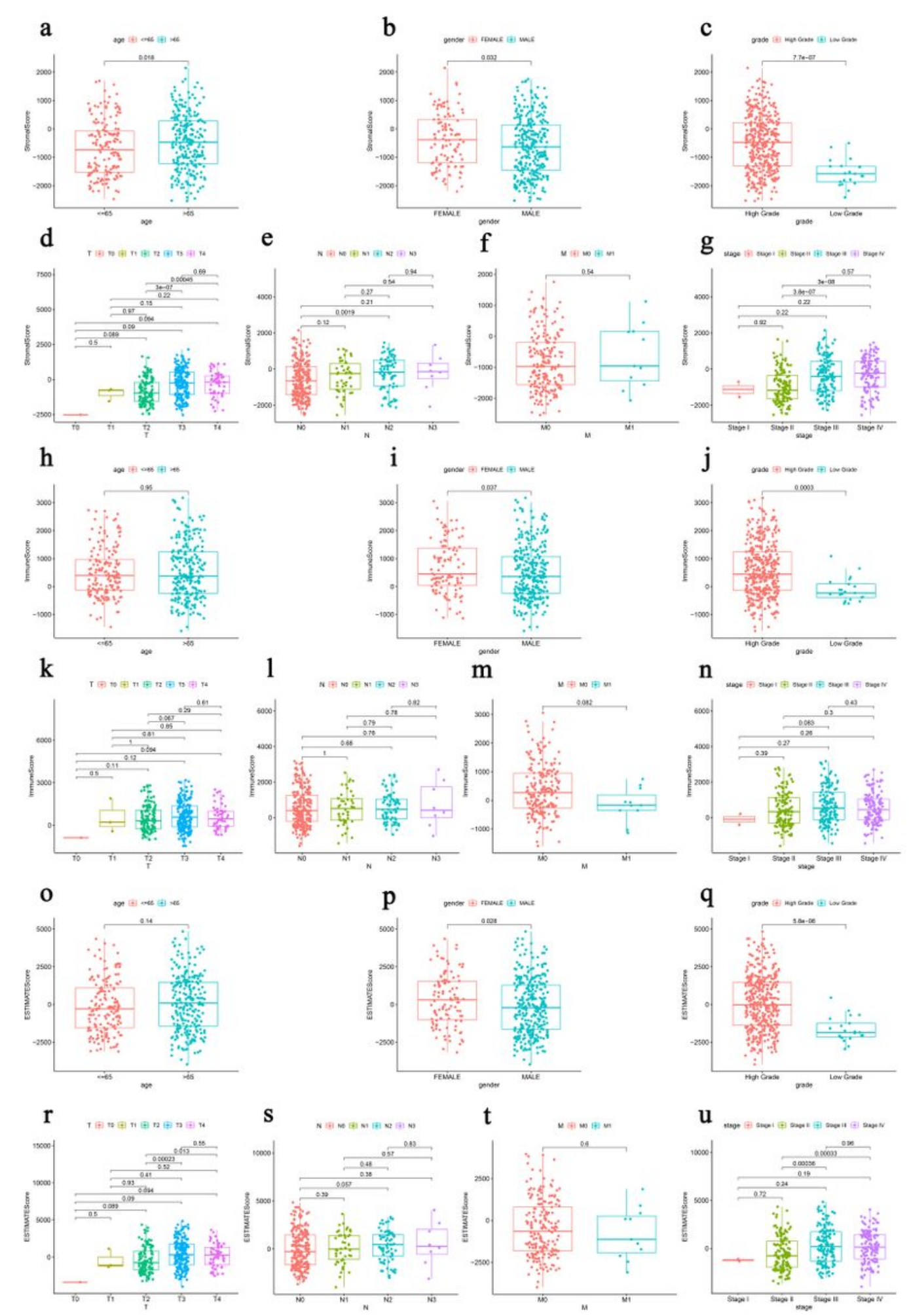

\section{Figure 2}

The relationship between Scores and clinic-pathological characteristics of BC patients. (a-g) The relationship between Stromal Score and clinic-pathological characteristics of BC patients, including age, gender, grade, TNM classification and stage; (h-n) The relationship between Immune Score and clinicpathological characteristics of BC patients; (o-u) The relationship between ESTIMATE Score and clinicpathological characteristics of BC patients. P-value was showed in each plot. 

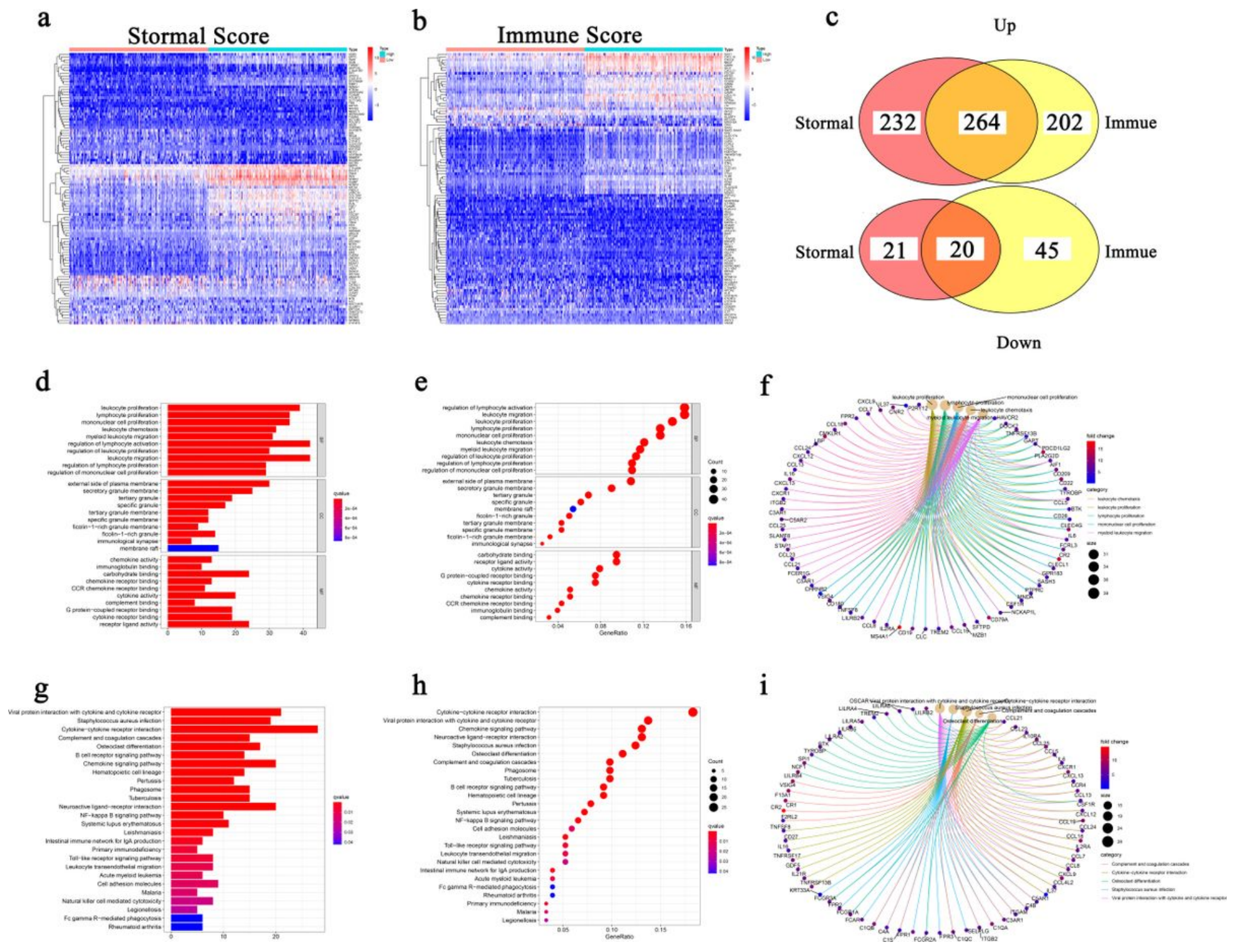

\section{Figure 3}

The identification and enrichment analysis of DEGs. (a-b) The heatmaps of DEGs obtained by comparison between high Stromal Score samples and low Stromal Score samples (or high Immune Score samples and low Immune Score). p $<0.05, F D R<0.05$ and log2FC $>2$ were set up to scan the DEGs; (c) Venn plots of DEGs which were commonly up-regulated or down-regulated in both Immune and Stromal Score; (d-f) GO enrichment analysis of 284 DEGs; $p<0.05$ was considered to be statistically significant; BP: biological process; CC: cell component; MF: molecular function; (g-i) KEGG enrichment analysis of 284 DEGs; $p<0.05$ was considered to be statistically significant; 

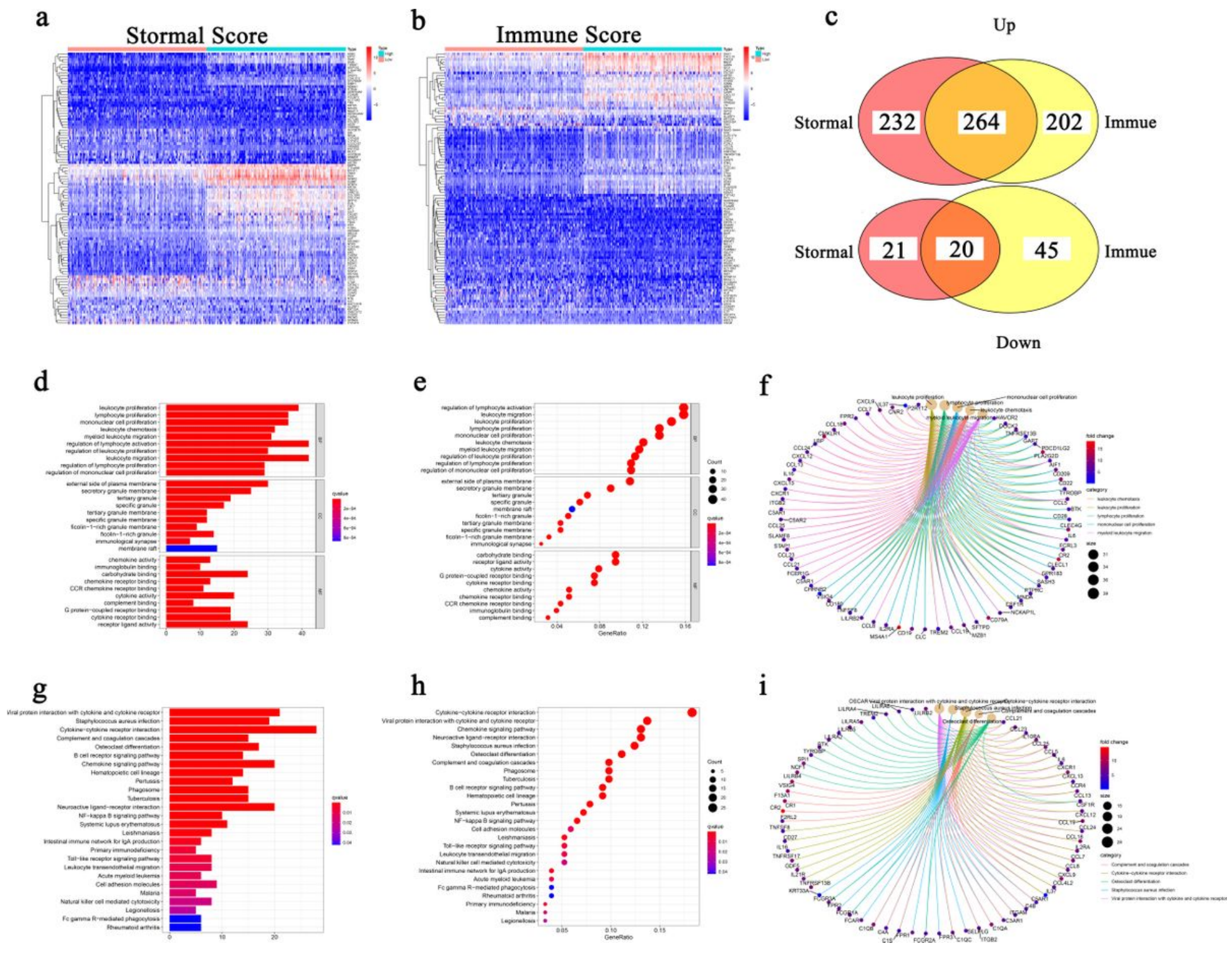

\section{Figure 3}

The identification and enrichment analysis of DEGs. (a-b) The heatmaps of DEGs obtained by comparison between high Stromal Score samples and low Stromal Score samples (or high Immune Score samples and low Immune Score). p $<0.05, F D R<0.05$ and log2FC $>2$ were set up to scan the DEGs; (c) Venn plots of DEGs which were commonly up-regulated or down-regulated in both Immune and Stromal Score; (d-f) GO enrichment analysis of 284 DEGs; $p<0.05$ was considered to be statistically significant; BP: biological process; CC: cell component; MF: molecular function; (g-i) KEGG enrichment analysis of 284 DEGs; $p<0.05$ was considered to be statistically significant; 

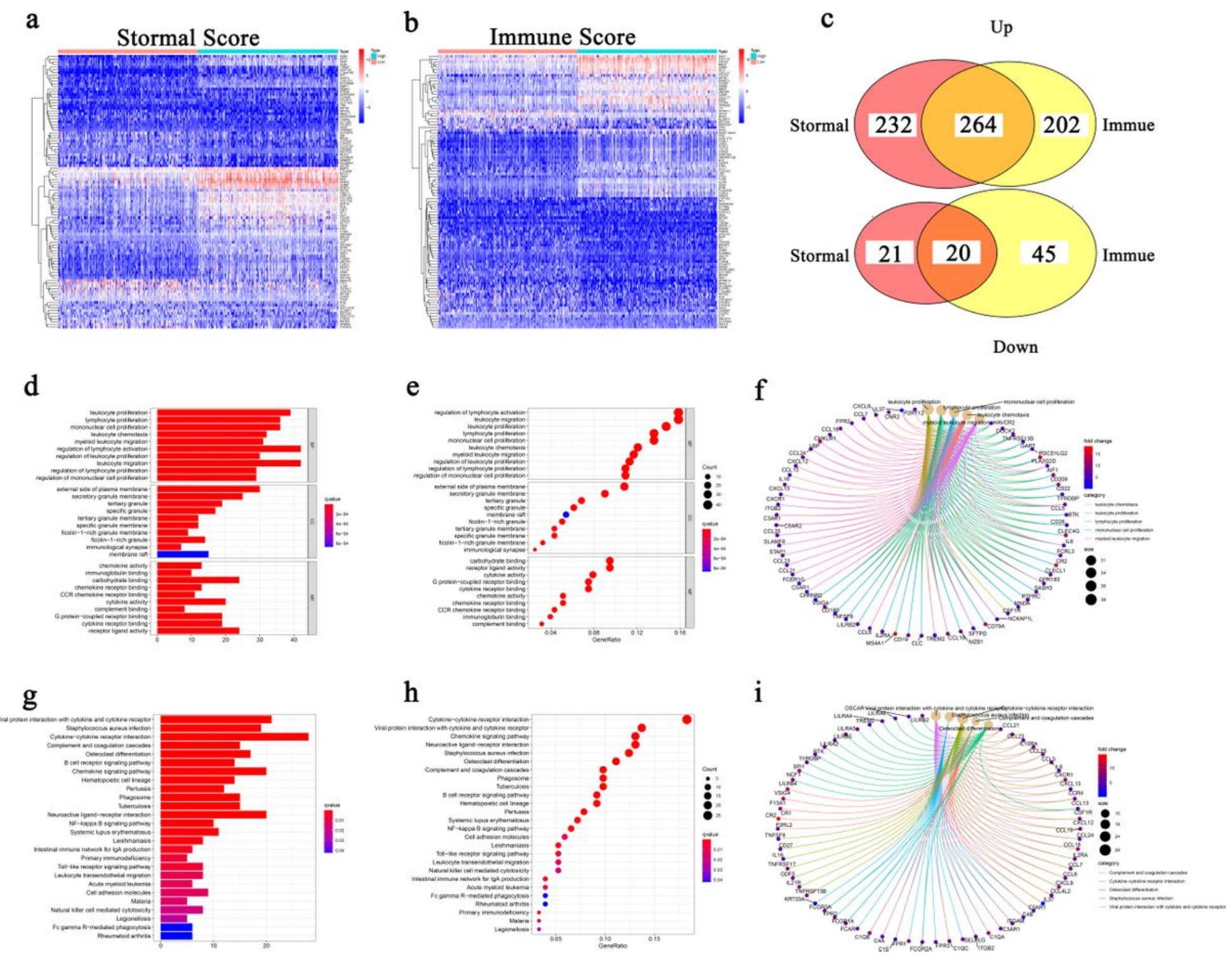

\section{Figure 3}

The identification and enrichment analysis of DEGs. (a-b) The heatmaps of DEGs obtained by comparison between high Stromal Score samples and low Stromal Score samples (or high Immune Score samples and low Immune Score). p $<0.05, F D R<0.05$ and log2FC $>2$ were set up to scan the DEGs; (c) Venn plots of DEGs which were commonly up-regulated or down-regulated in both Immune and Stromal Score; (d-f) GO enrichment analysis of 284 DEGs; $p<0.05$ was considered to be statistically significant; BP: biological process; CC: cell component; MF: molecular function; (g-i) KEGG enrichment analysis of 284 DEGs; $p<0.05$ was considered to be statistically significant; 
a

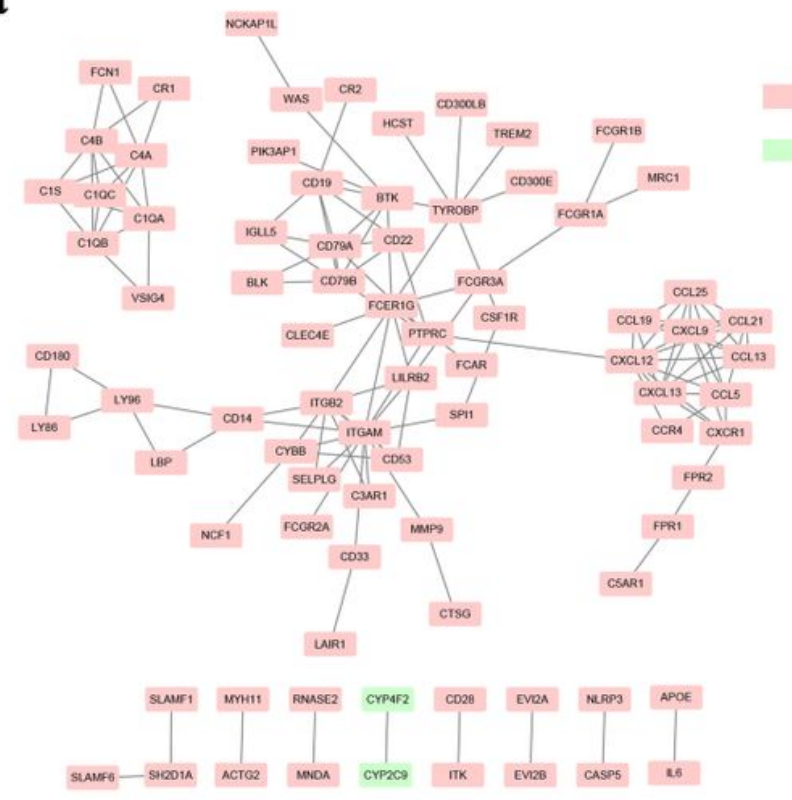

C

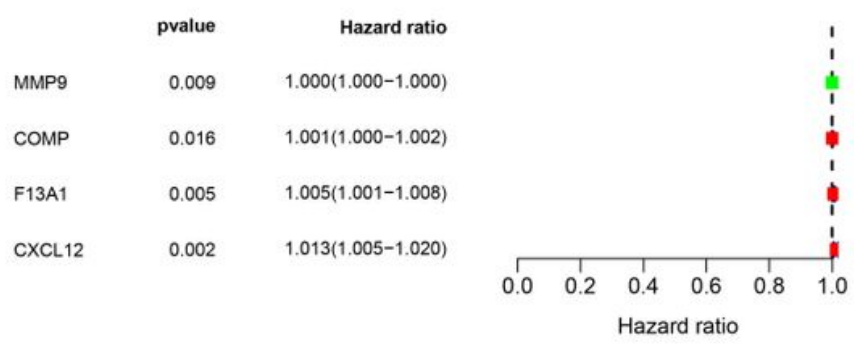

b

up-regulated

down-regulated

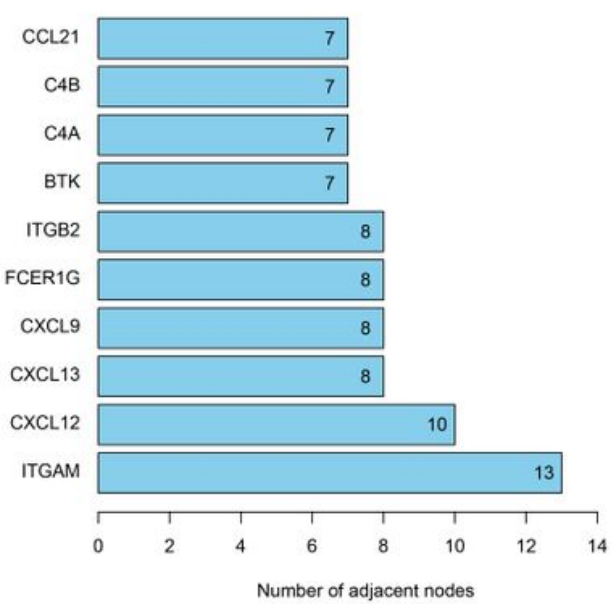

d

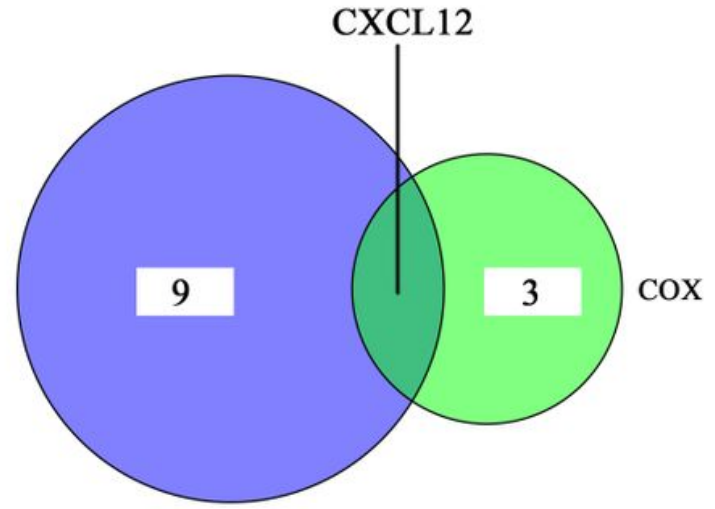

\section{Figure 4}

PPI network and univariate COX regression analysis of 284 DEGs. (a) PPI network was constructed by STRING database and Cytoscape software; interaction confidence value >0.95; (b) The top 10 genes which shared the leading nodes in PPI network; (c) Univariate COX regression analysis of 284 DEGs; $p<0.05$ was considered to be statistically significant; (d) Intersection analysis of DEGs which shared the leading nodes in PPI network and were closely related with BC patients' survival. $p<0.05$ was considered to be statistically significant. 
a

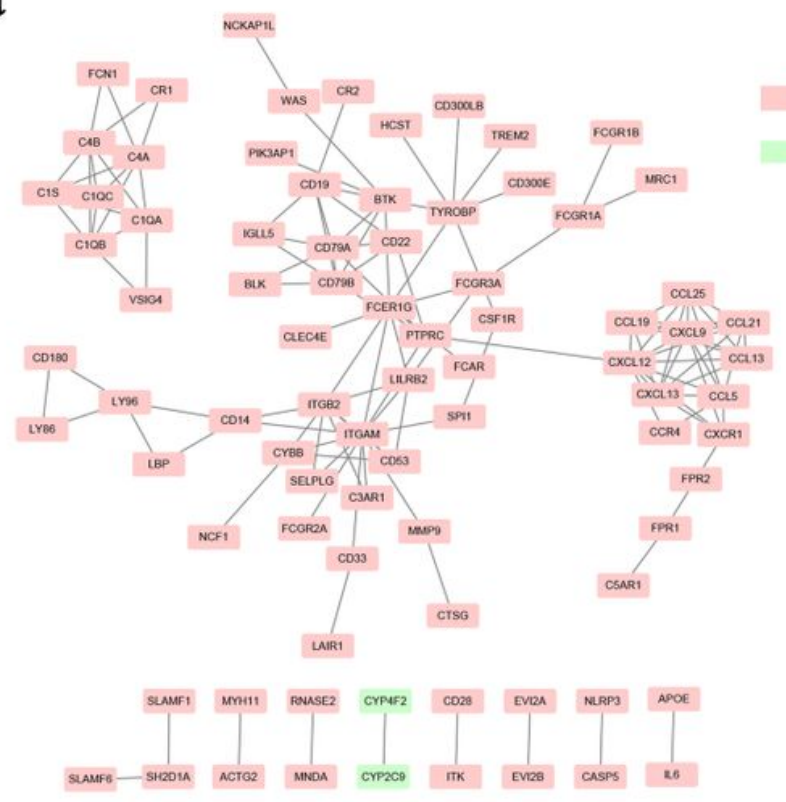

c

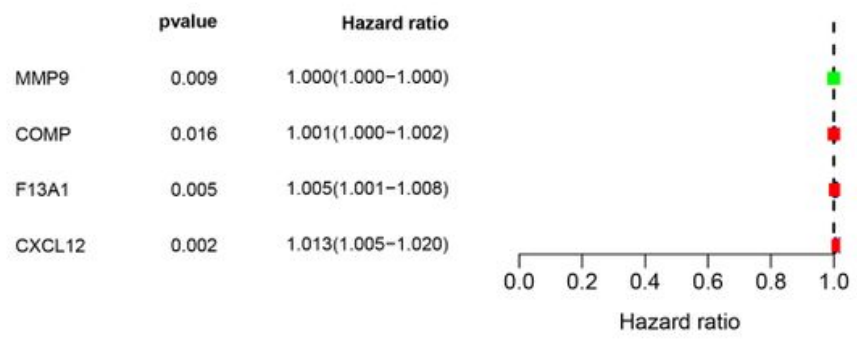

b

up-regulated

down-regulated

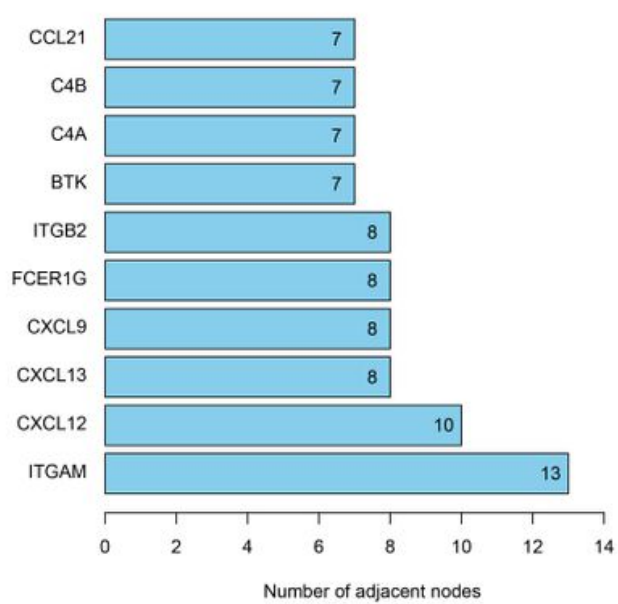

d

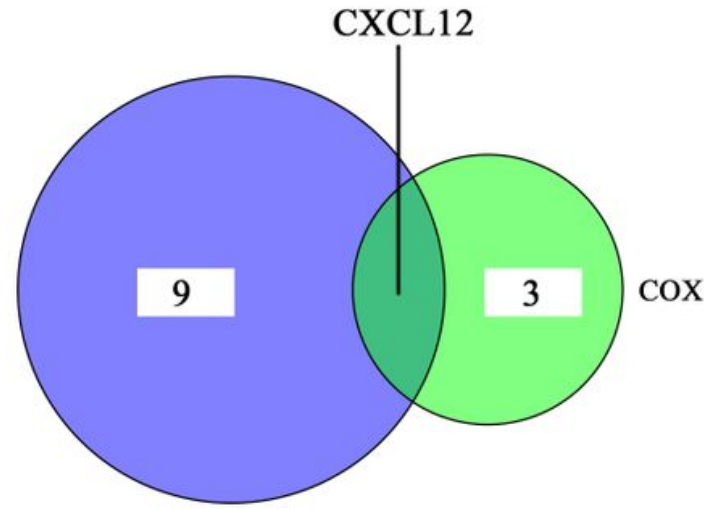

\section{Figure 4}

PPI network and univariate COX regression analysis of 284 DEGs. (a) PPI network was constructed by STRING database and Cytoscape software; interaction confidence value >0.95; (b) The top 10 genes which shared the leading nodes in PPI network; (c) Univariate COX regression analysis of 284 DEGs; $p<0.05$ was considered to be statistically significant; (d) Intersection analysis of DEGs which shared the leading nodes in PPI network and were closely related with BC patients' survival. $p<0.05$ was considered to be statistically significant. 
a

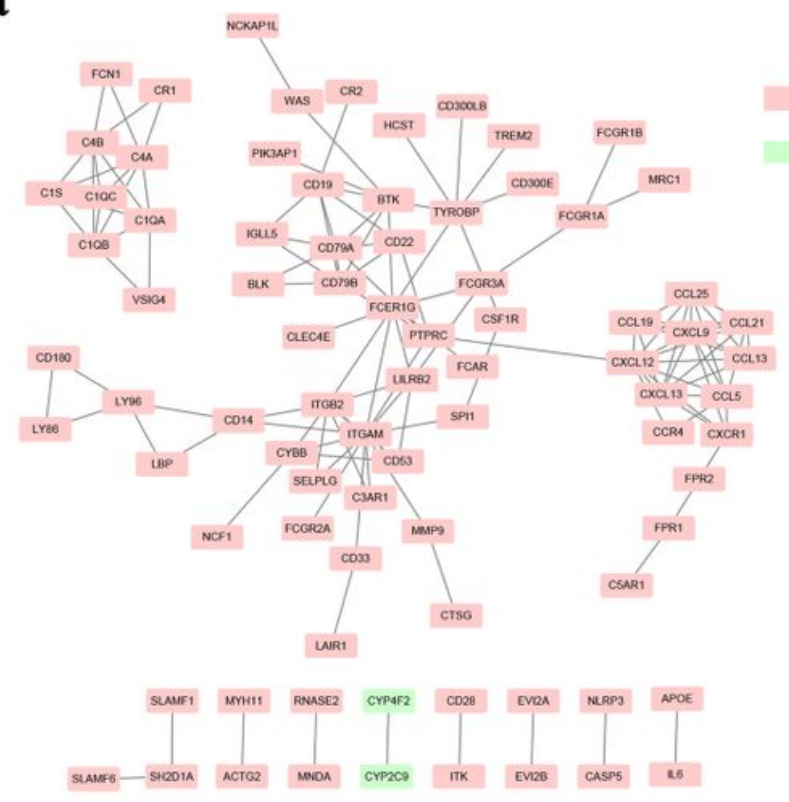

C

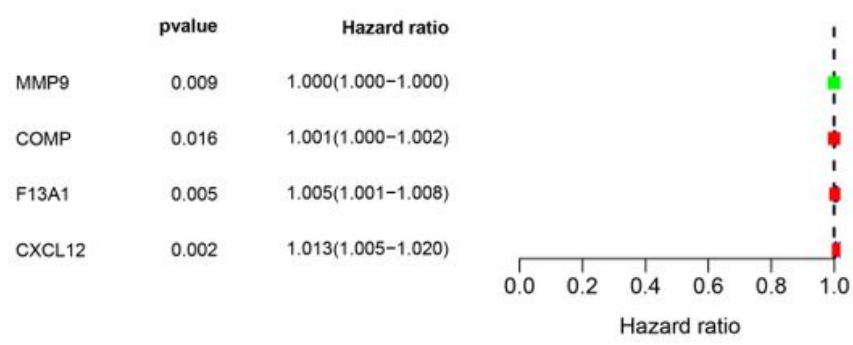

b

up-regulated

down-regulated

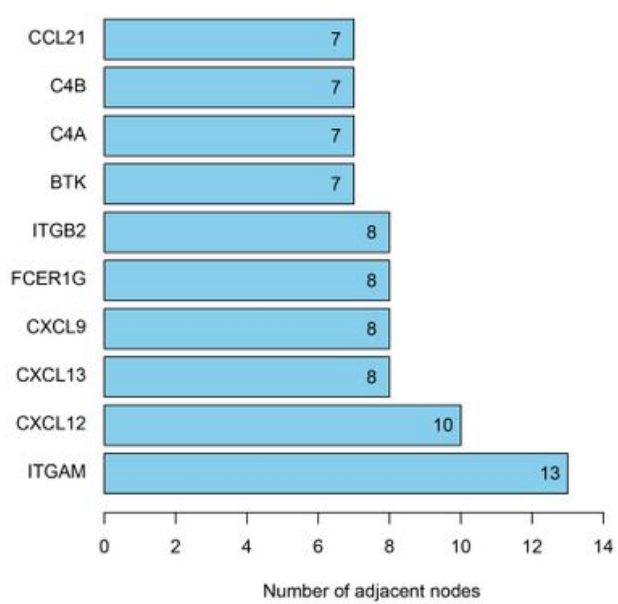

d

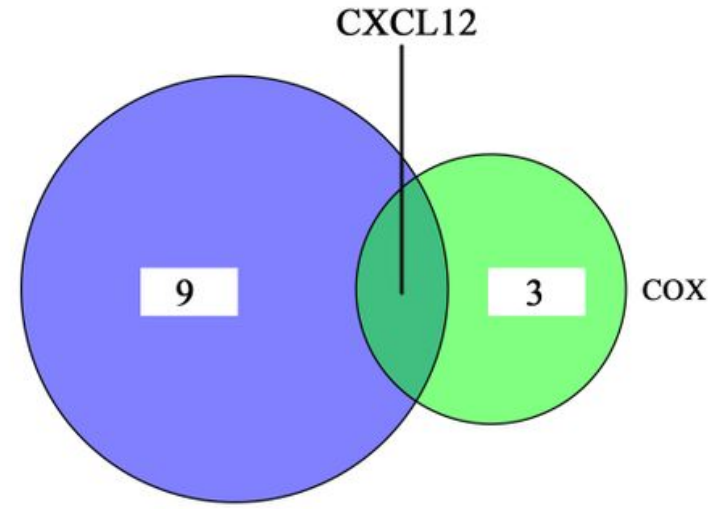

\section{Figure 4}

PPI network and univariate COX regression analysis of 284 DEGs. (a) PPI network was constructed by STRING database and Cytoscape software; interaction confidence value >0.95; (b) The top 10 genes which shared the leading nodes in PPI network; (c) Univariate COX regression analysis of 284 DEGs; $p<0.05$ was considered to be statistically significant; (d) Intersection analysis of DEGs which shared the leading nodes in PPI network and were closely related with BC patients' survival. $p<0.05$ was considered to be statistically significant. 
a

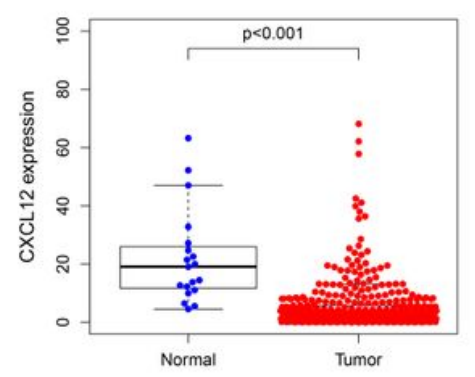

d

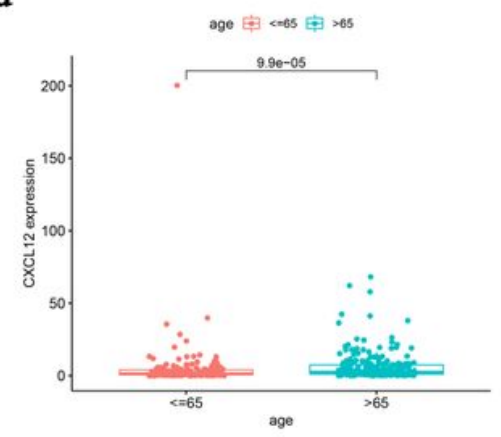

b

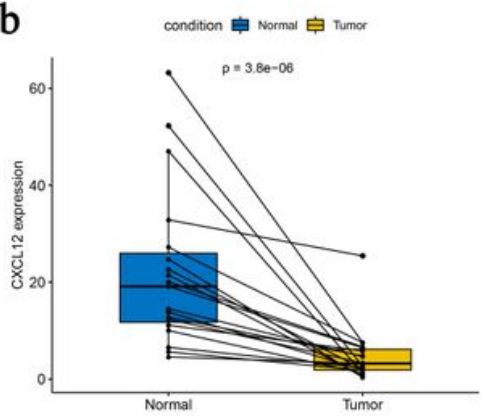

e

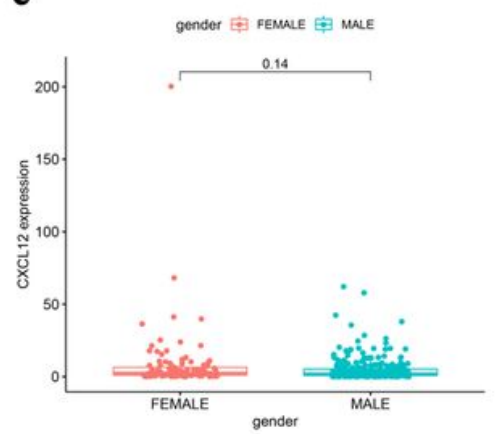

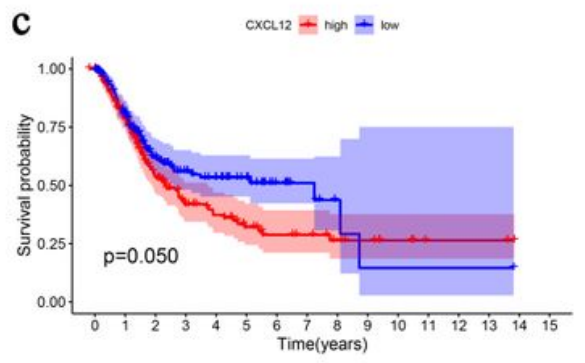

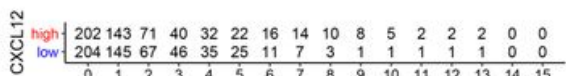

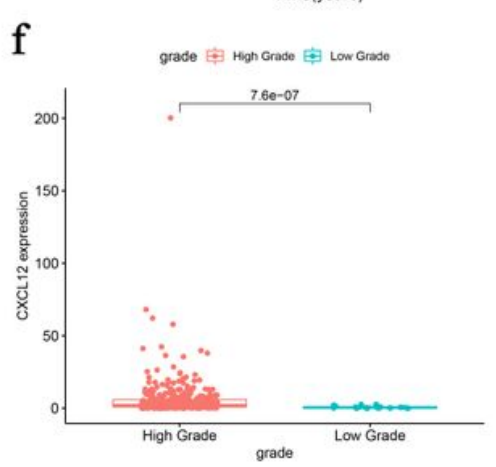

$\mathrm{h}$

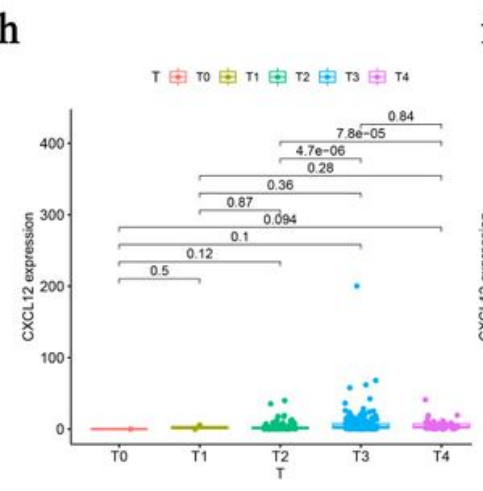

i

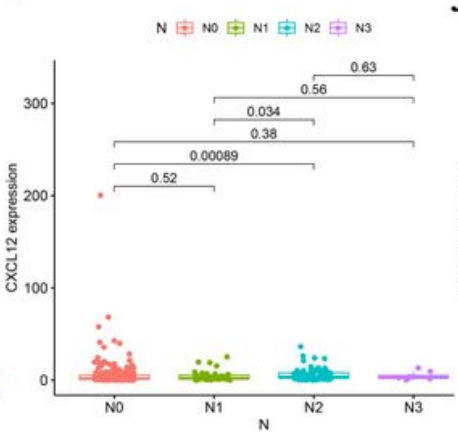

j

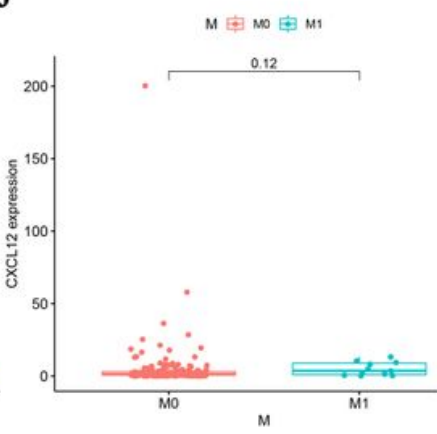

$\mathbf{k}$

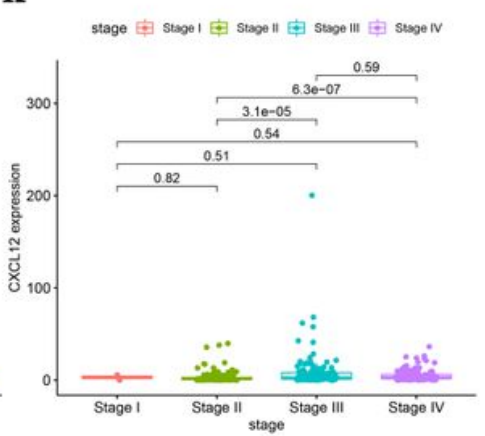

\section{Figure 5}

Single gene analysis of CXCL12 in BC. (a) Difference analysis of the expression of CXCL12 between BC samples and normal samples; (b) Paired analysis of the expression of CXCL12 between BC samples and paired normal samples; (c) Survival analysis of the expression of CXCL12 and the BC patients' survival; patients were grouped into high CXCL12 group and low CXCL12 group, compared with the median; $p$ value and the number of samples were displayed in the diagram; $(d-k)$ The relationship between the expression of CXCL12 and clinic-pathological characteristics of BC patients, including age, gender, grade, TNM classification and stage; $p$ value was displayed in the diagram and $p<0.05$ was considered to be statistically significant. 
a

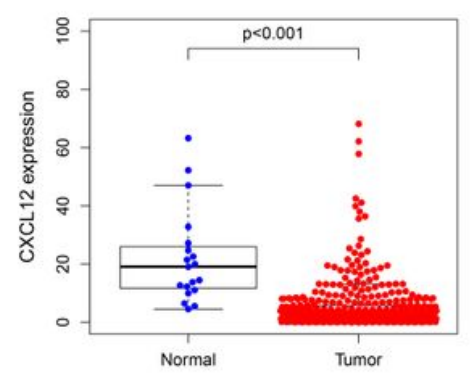

d

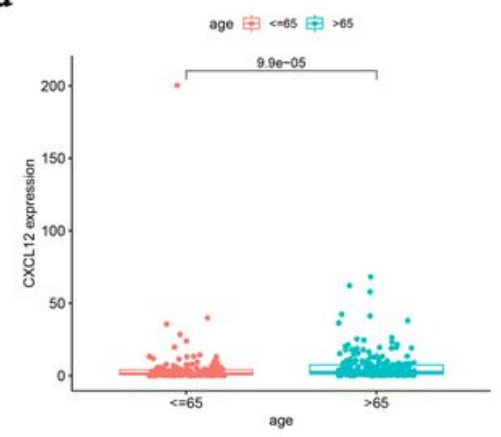

b

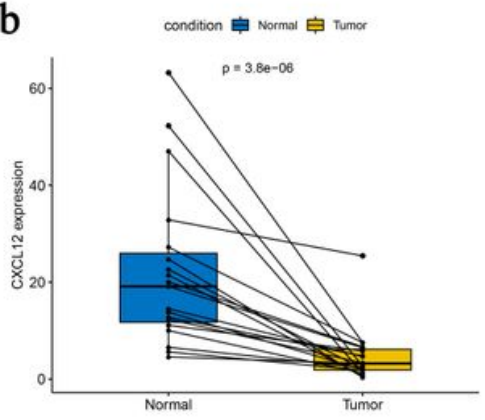

e

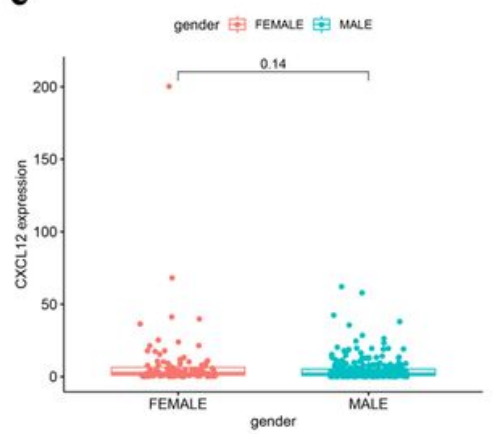

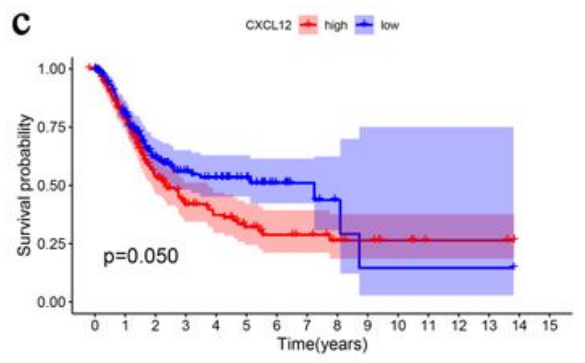

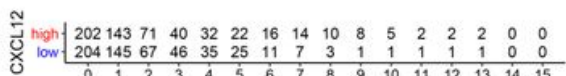

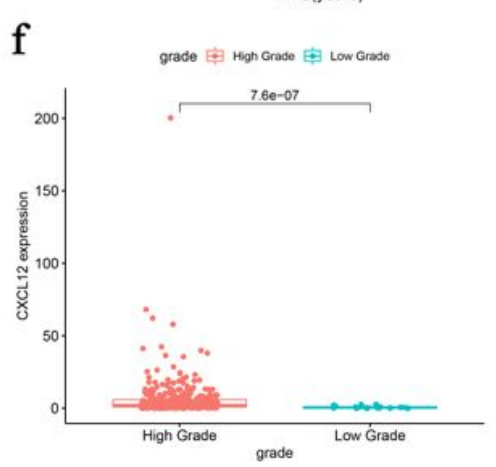

$\mathrm{h}$

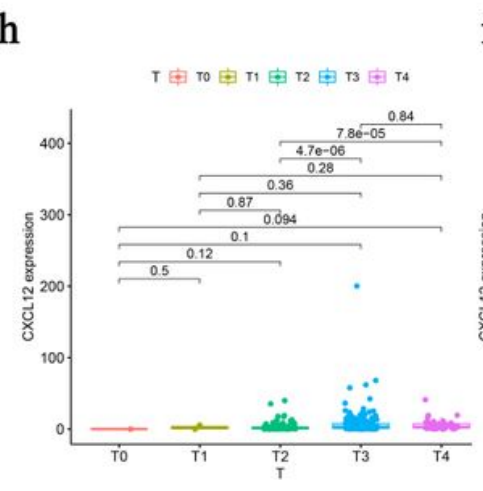

i

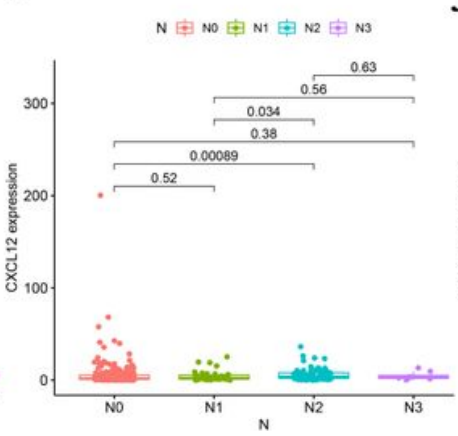

j

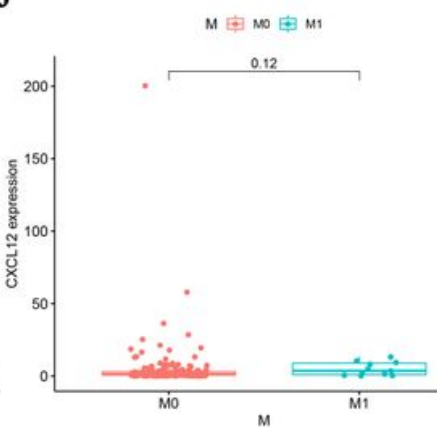

$\mathbf{k}$

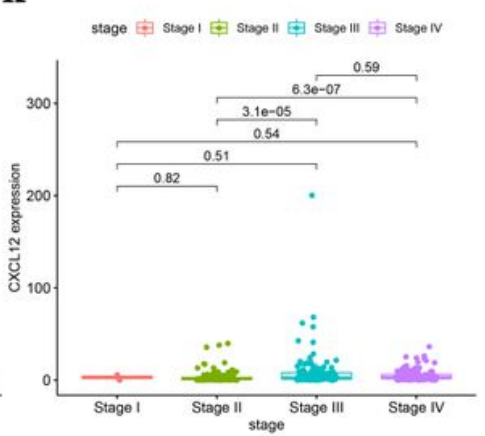

\section{Figure 5}

Single gene analysis of CXCL12 in BC. (a) Difference analysis of the expression of CXCL12 between BC samples and normal samples; (b) Paired analysis of the expression of CXCL12 between BC samples and paired normal samples; (c) Survival analysis of the expression of CXCL12 and the BC patients' survival; patients were grouped into high CXCL12 group and low CXCL12 group, compared with the median; $p$ value and the number of samples were displayed in the diagram; $(d-k)$ The relationship between the expression of CXCL12 and clinic-pathological characteristics of BC patients, including age, gender, grade, TNM classification and stage; $p$ value was displayed in the diagram and $p<0.05$ was considered to be statistically significant. 
a

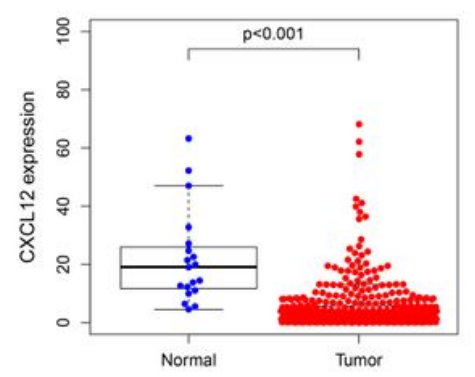

d

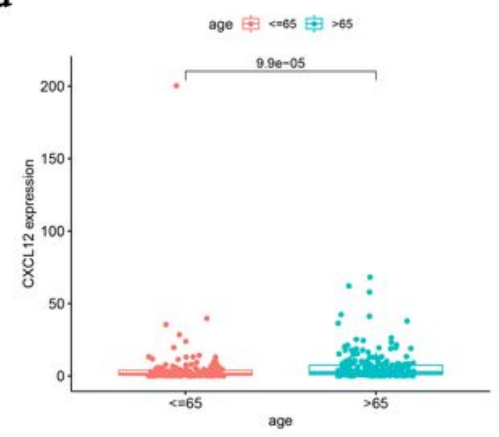

b

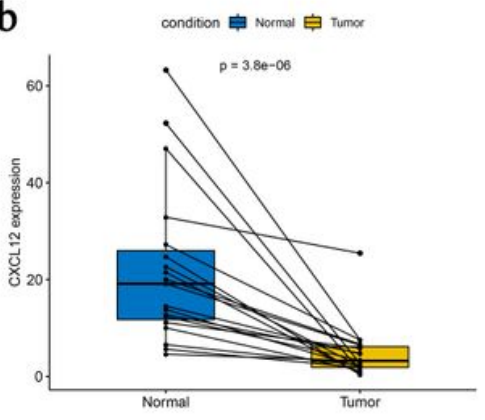

e

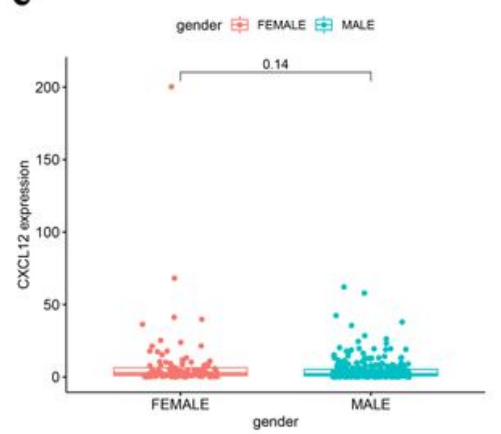

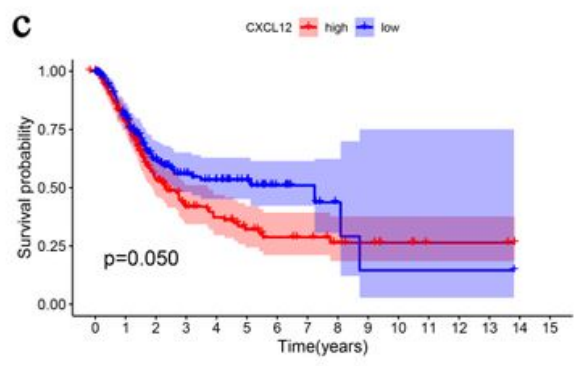

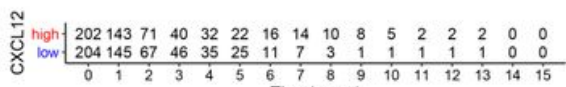

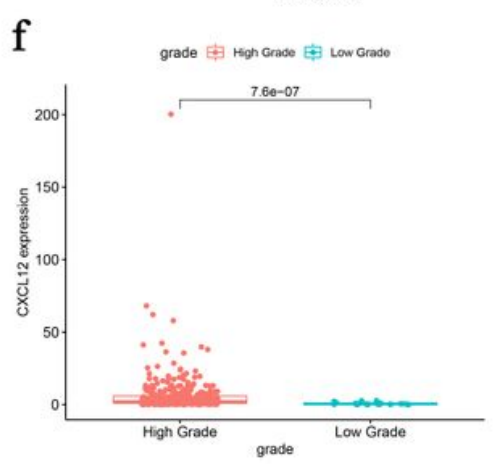

h

i

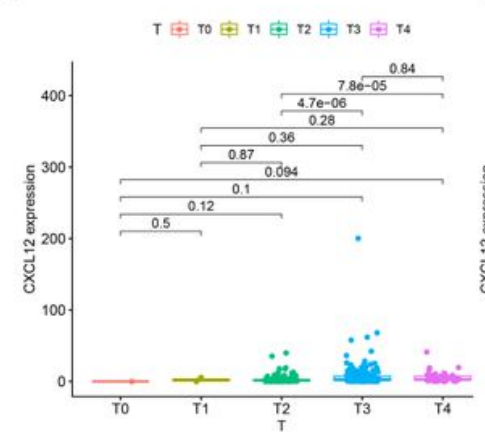

i

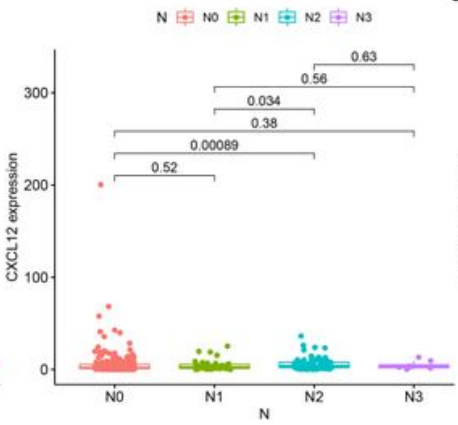

j

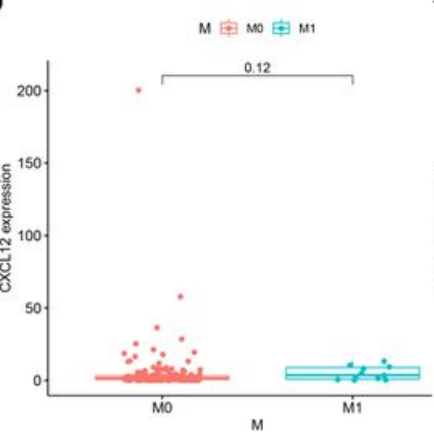

$\mathrm{k}$

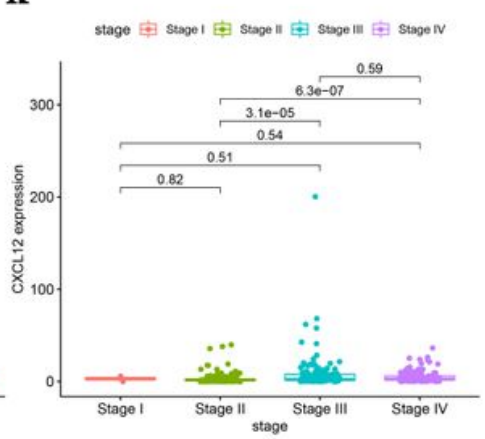

\section{Figure 5}

Single gene analysis of CXCL12 in BC. (a) Difference analysis of the expression of CXCL12 between BC samples and normal samples; (b) Paired analysis of the expression of CXCL12 between BC samples and paired normal samples; (c) Survival analysis of the expression of CXCL12 and the BC patients' survival; patients were grouped into high CXCL12 group and low CXCL12 group, compared with the median; $p$ value and the number of samples were displayed in the diagram; $(d-k)$ The relationship between the expression of CXCL12 and clinic-pathological characteristics of BC patients, including age, gender, grade, TNM classification and stage; $p$ value was displayed in the diagram and $p<0.05$ was considered to be statistically significant. 

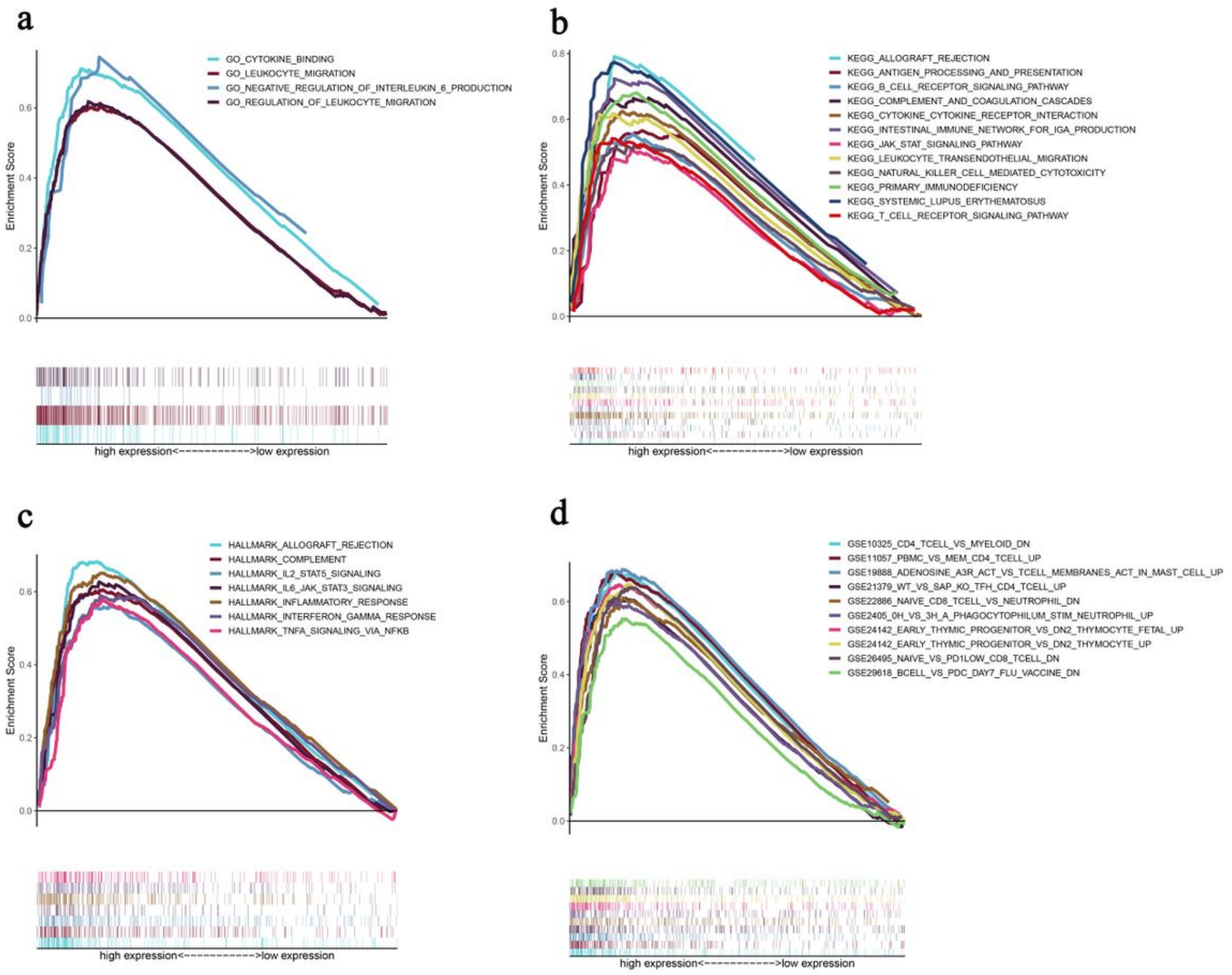

Figure 6

GSEA for genes in high CXCL12 expression group. (a) GSEA for genes in high CXCL12 expression group in $\mathrm{C} 5$ collection, the gene ontology sets; (b) GSEA for genes in high CXCL12 expression group in C2 collection, the KEGG gene sets database; (c) GSEA for genes in high CXCL12 expression group in hallmark gene sets; (d) GSEA for genes in high CXCL12 expression group in C7 collection defined by MSigDB, the immunologic gene sets. FDR $<0.05$ and $p<0.05$ were considered to be statistically significant. 

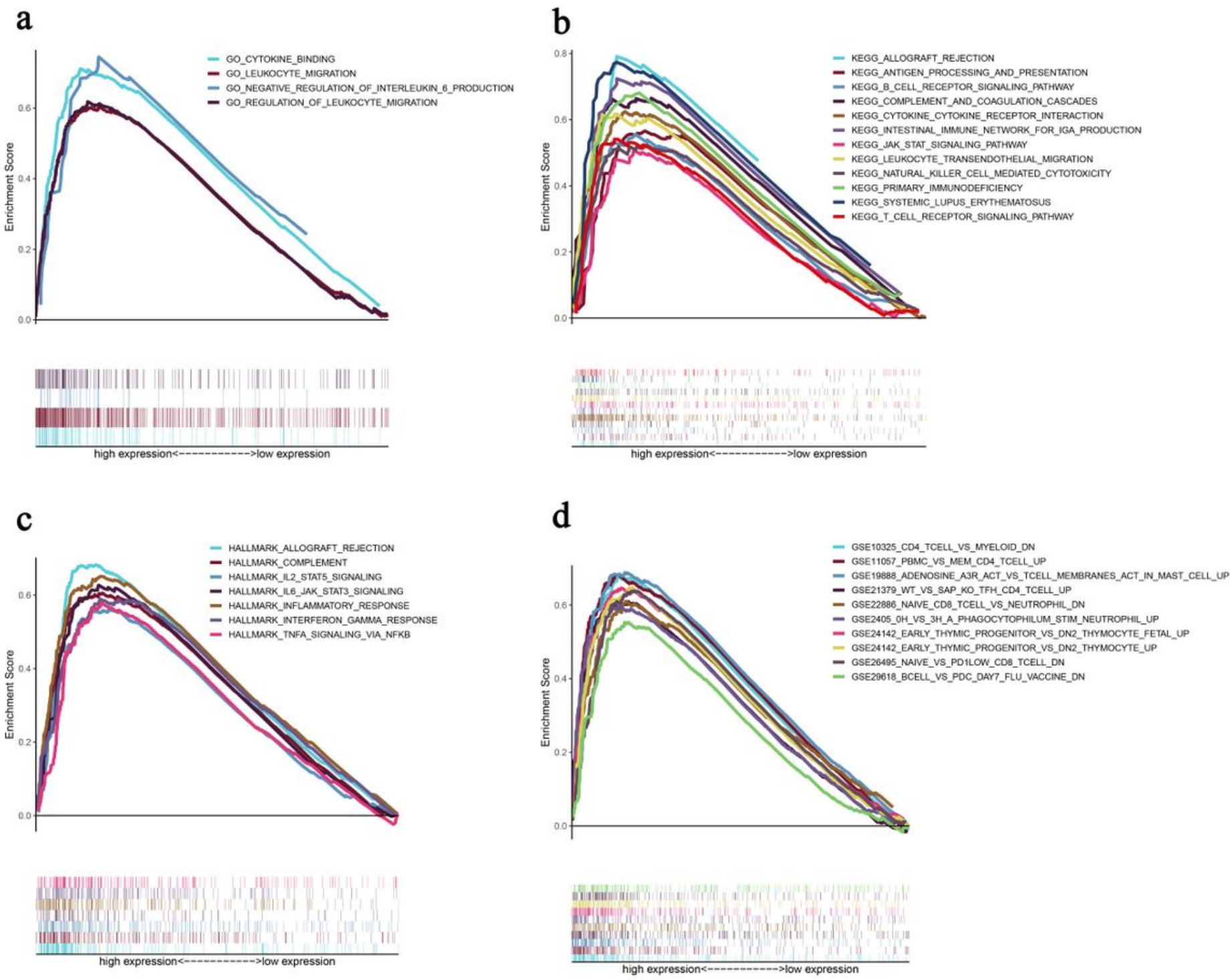

Figure 6

GSEA for genes in high CXCL12 expression group. (a) GSEA for genes in high CXCL12 expression group in $\mathrm{C} 5$ collection, the gene ontology sets; (b) GSEA for genes in high CXCL12 expression group in C2 collection, the KEGG gene sets database; (c) GSEA for genes in high CXCL12 expression group in hallmark gene sets; (d) GSEA for genes in high CXCL12 expression group in C7 collection defined by MSigDB, the immunologic gene sets. FDR $<0.05$ and $p<0.05$ were considered to be statistically significant. 

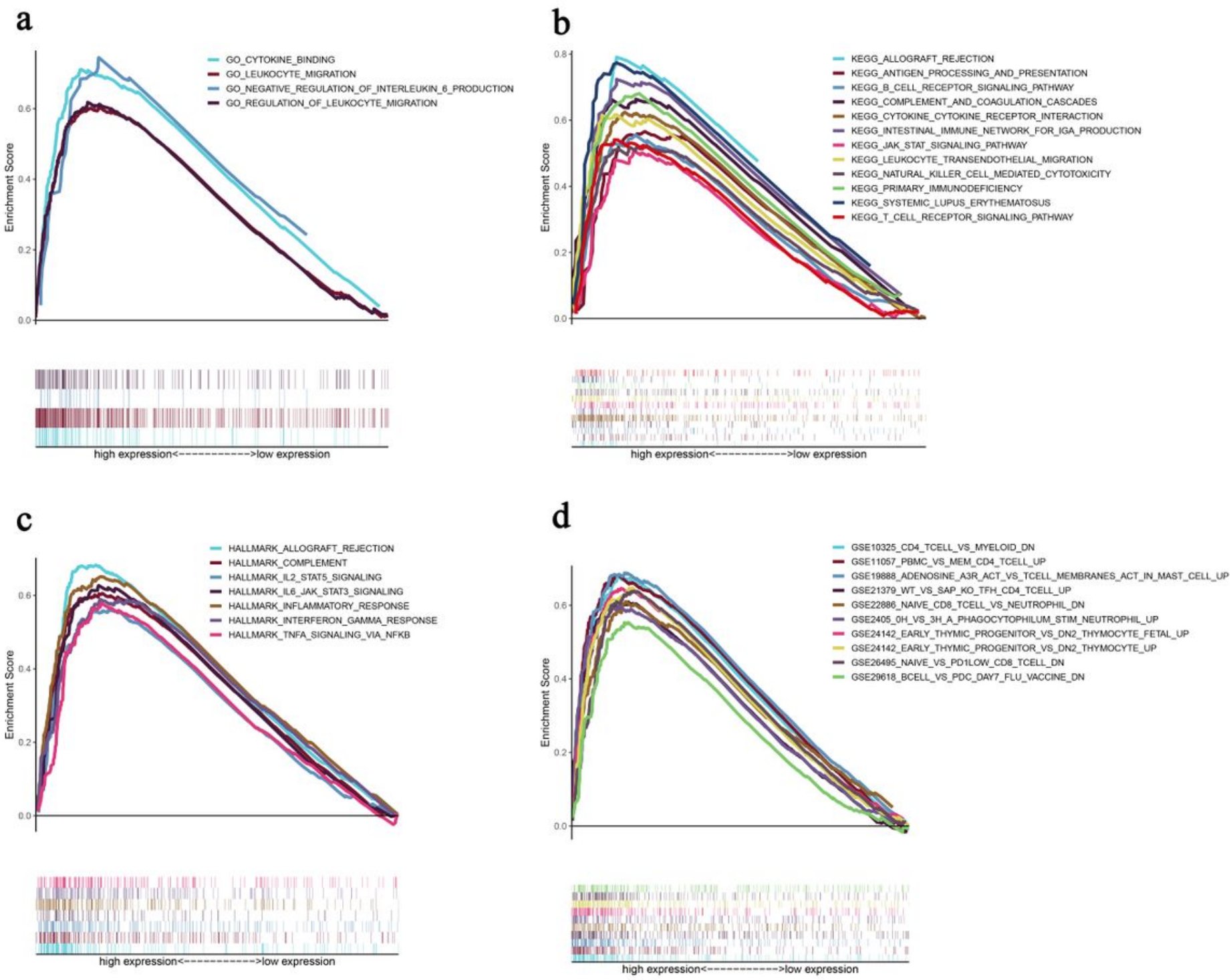

Figure 6

GSEA for genes in high CXCL12 expression group. (a) GSEA for genes in high CXCL12 expression group in $\mathrm{C} 5$ collection, the gene ontology sets; (b) GSEA for genes in high CXCL12 expression group in C2 collection, the KEGG gene sets database; (c) GSEA for genes in high CXCL12 expression group in hallmark gene sets; (d) GSEA for genes in high CXCL12 expression group in C7 collection defined by MSigDB, the immunologic gene sets. FDR $<0.05$ and $p<0.05$ were considered to be statistically significant. 


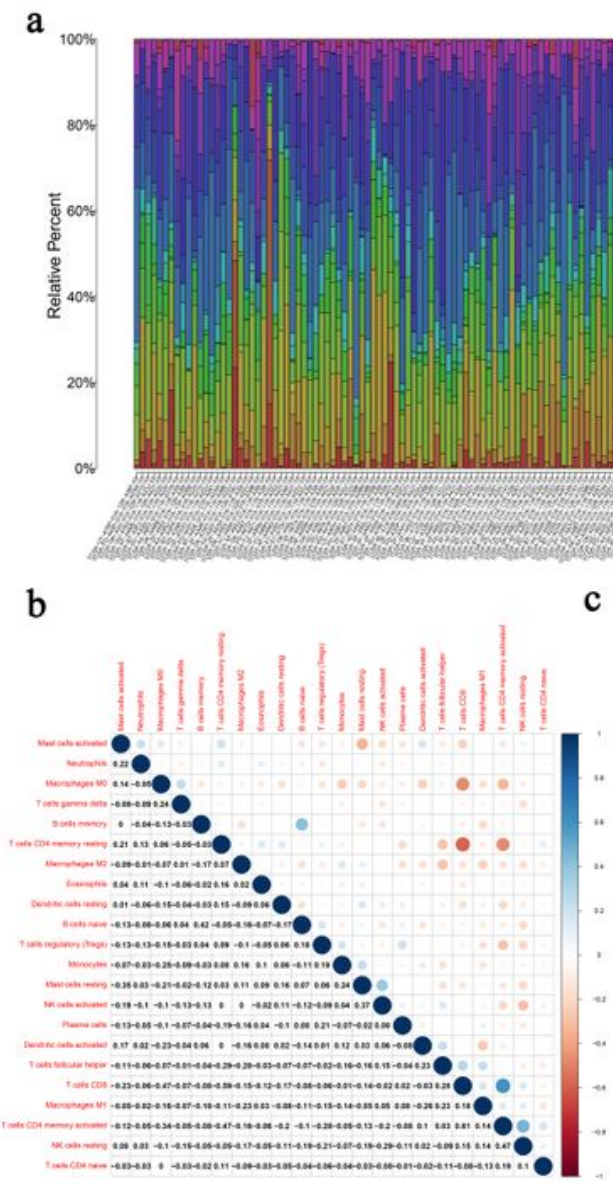

c
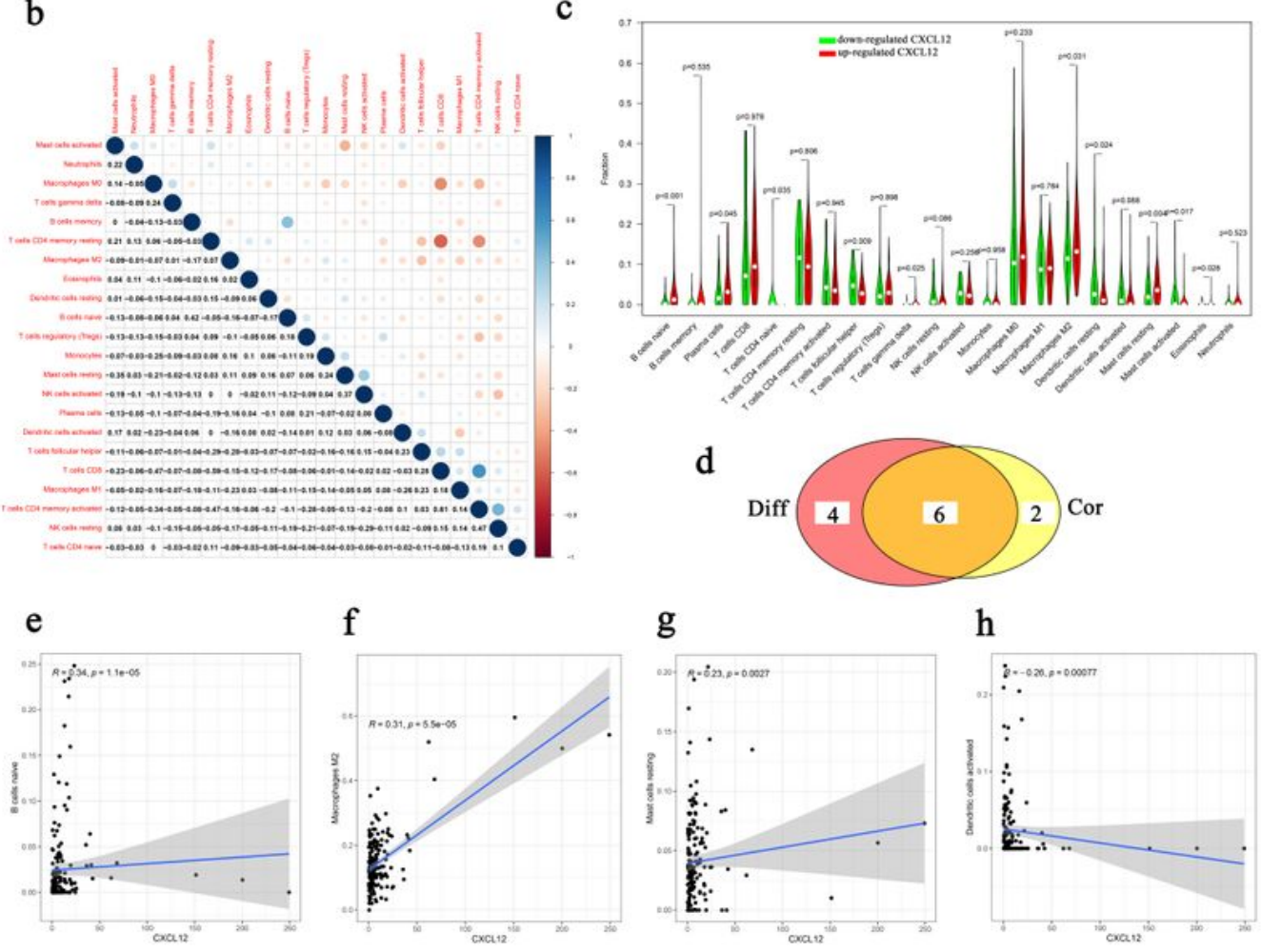

f

g

h
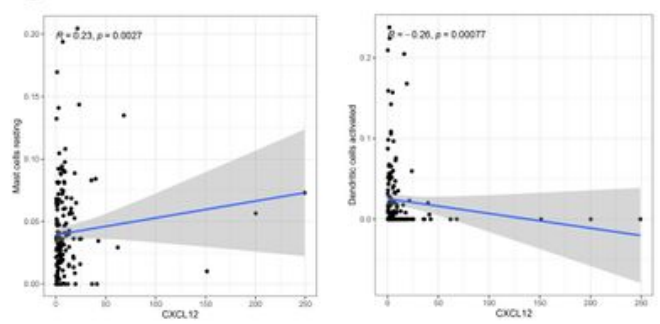

i

k

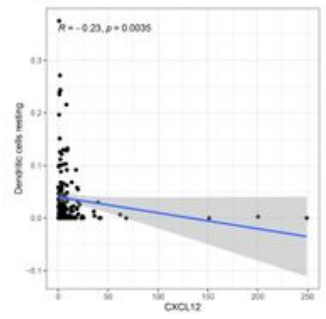

j
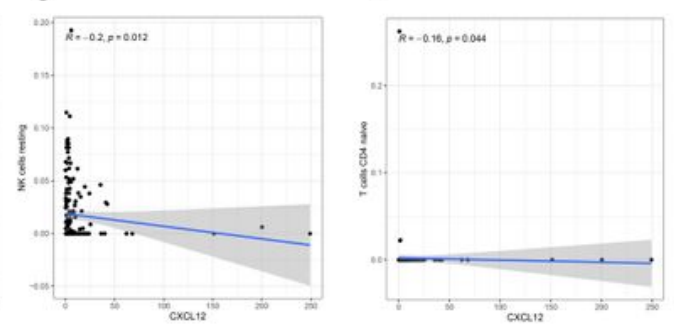

1

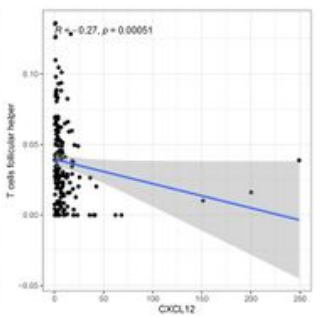

\section{Figure 7}

TICs Profile, and the difference analysis as well as correlation analysis between CXCL12 and TICs in TIME of BC. (a) Barplot displayed the ratio of 22 TICs in BC samples; (b) The correlation analysis among 22 TICs; each spot indicated p value; (c) Violin plot diaplayed the association between the expression of CXCL12 and 22 TICs; $p<0.05$ was considered to be statistically significant; (d) Venn plot showed 6 TICs 
shared by the difference test (c) and correlation test (e-l). (e-l) Scatter plots showed the association between the expression of CXCL12 and 8 TICs; $p<0.05$ was considered to be statistically significant.

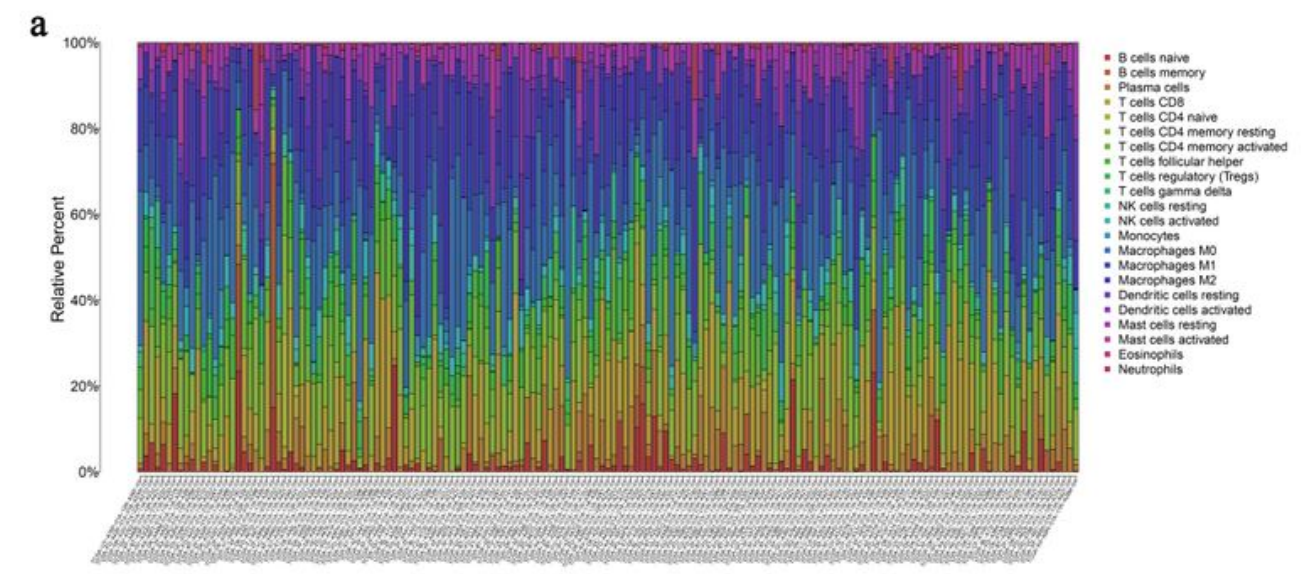

b
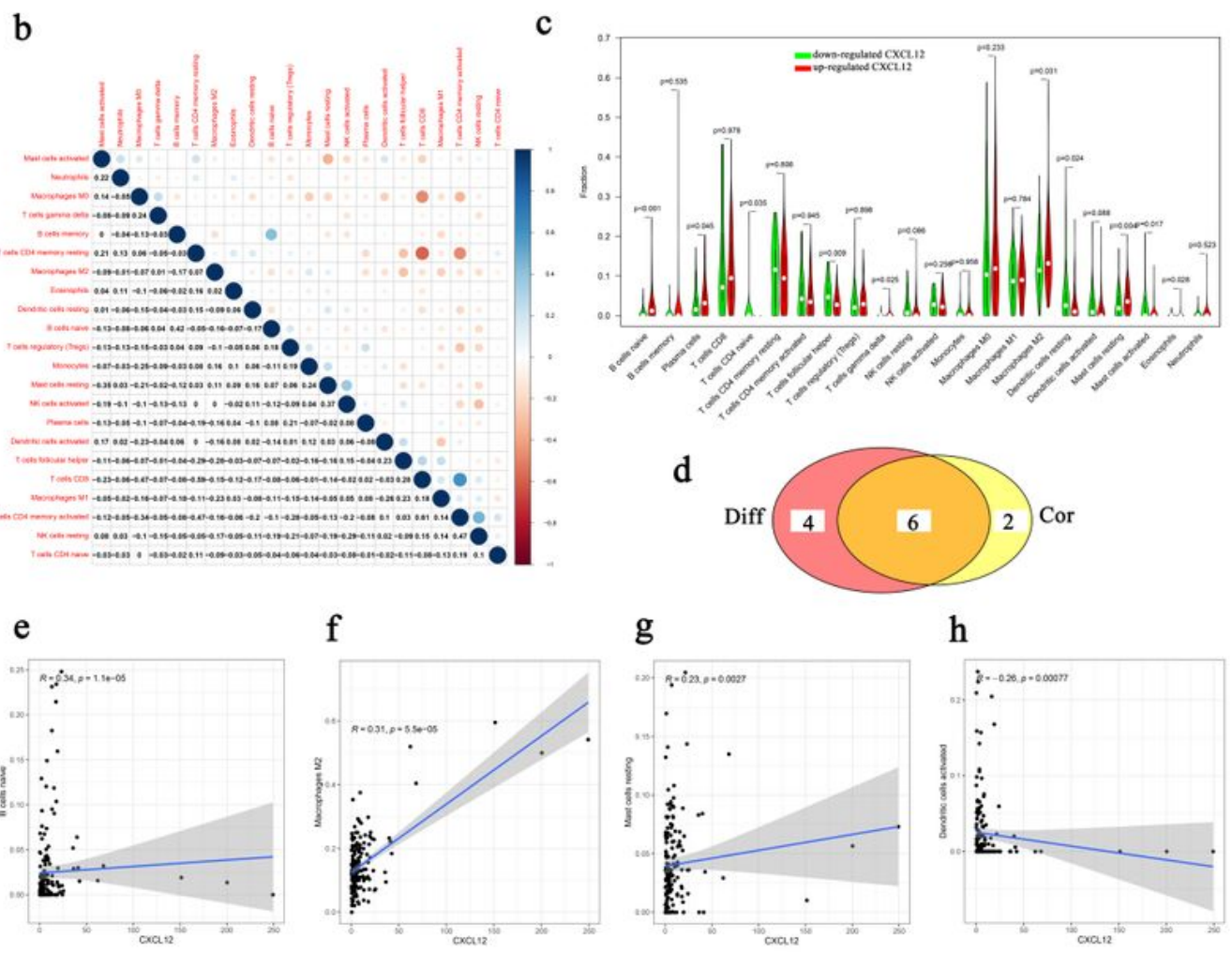

g

$\mathrm{h}$
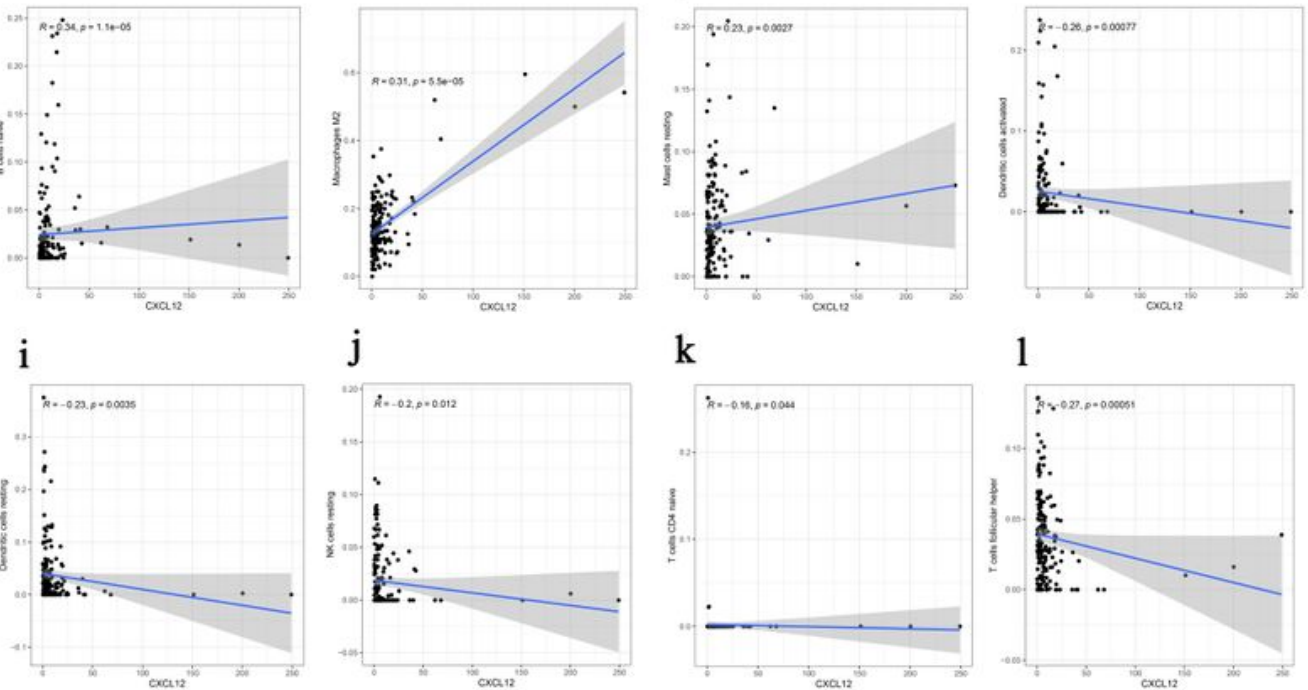

$\mathbf{k}$

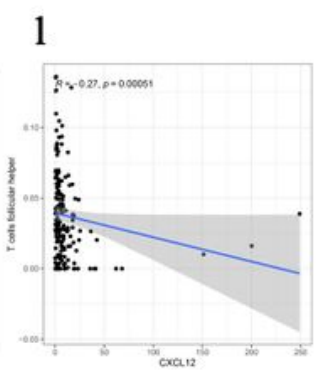

\section{Figure 7}

TICs Profile, and the difference analysis as well as correlation analysis between CXCL12 and TICs in TIME of BC. (a) Barplot displayed the ratio of 22 TICs in BC samples; (b) The correlation analysis among 22 TICs; each spot indicated p value; (c) Violin plot diaplayed the association between the expression of 
CXCL12 and 22 TICs; $p<0.05$ was considered to be statistically significant; (d) Venn plot showed 6 TICs shared by the difference test (c) and correlation test (e-l). (e-l) Scatter plots showed the association between the expression of CXCL12 and 8 TICs; $p<0.05$ was considered to be statistically significant.

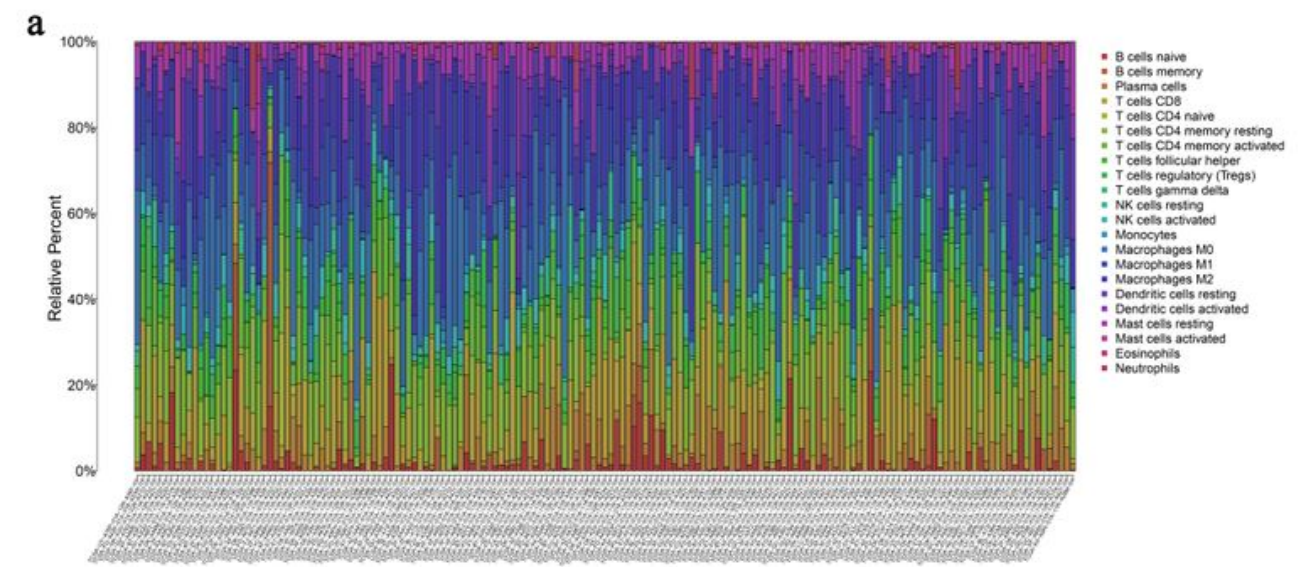

$$
\text { b }
$$

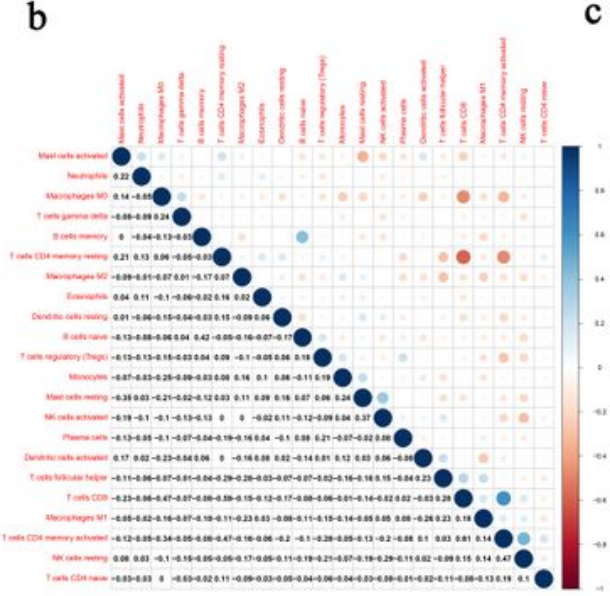

c

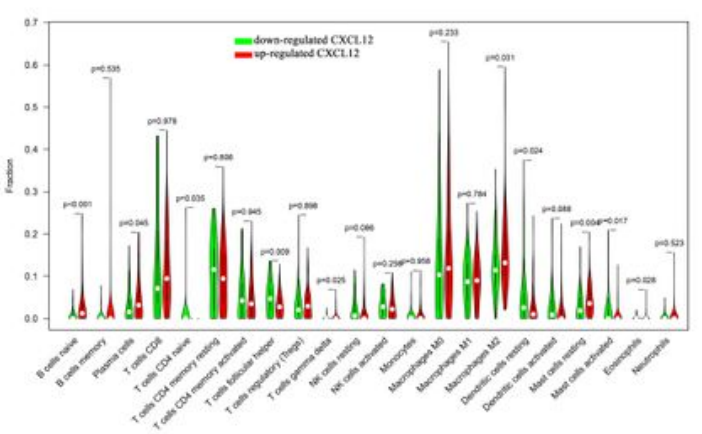

d
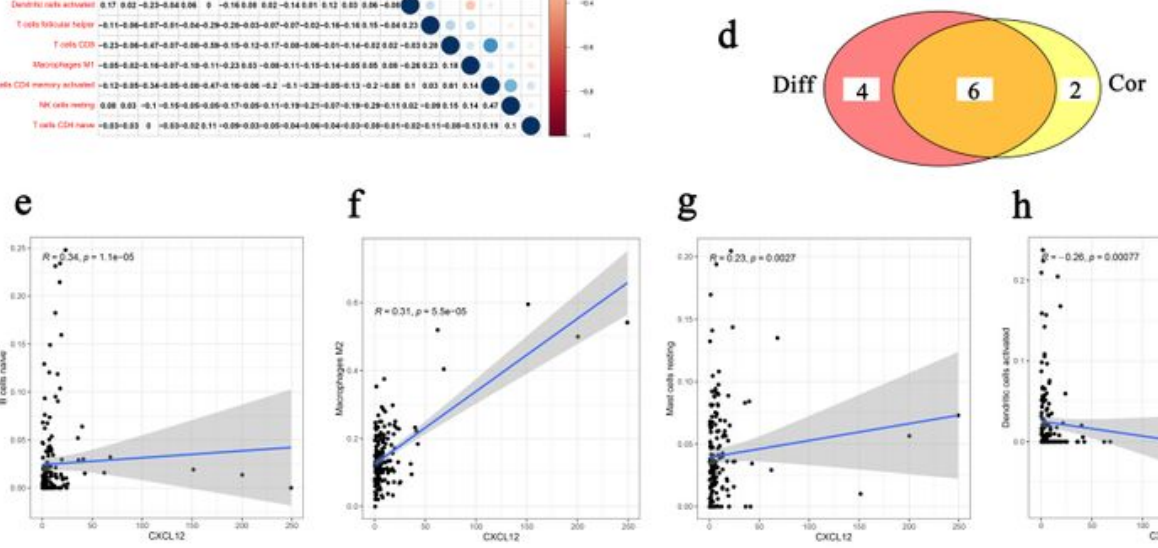

g

$\mathrm{h}$
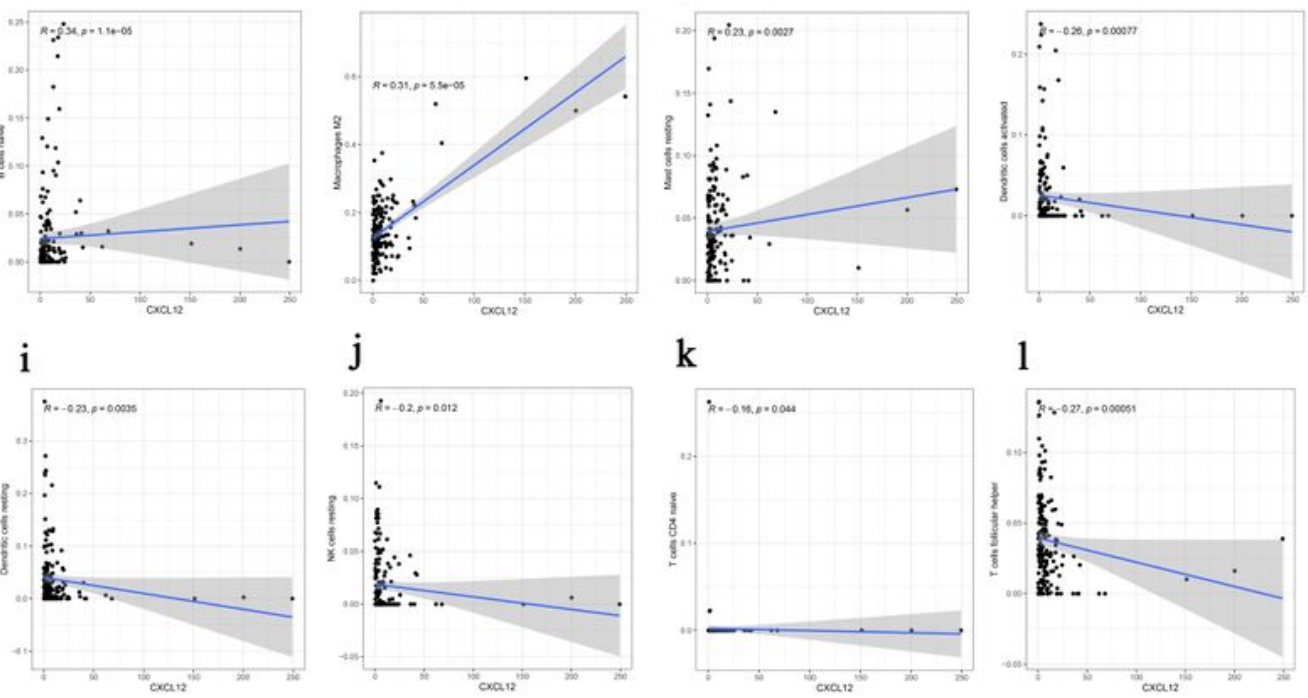

k

1
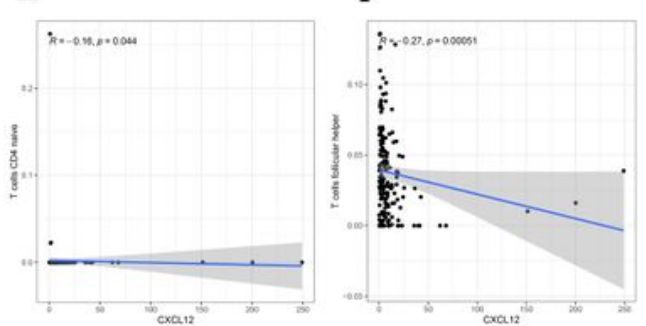

Figure 7

TICs Profile, and the difference analysis as well as correlation analysis between CXCL12 and TICs in TIME of BC. (a) Barplot displayed the ratio of 22 TICs in BC samples; (b) The correlation analysis among 
22 TICs; each spot indicated p value; (c) Violin plot diaplayed the association between the expression of CXCL12 and 22 TICs; $p<0.05$ was considered to be statistically significant; (d) Venn plot showed 6 TICs shared by the difference test (c) and correlation test (e-l). (e-l) Scatter plots showed the association between the expression of CXCL12 and 8 TICs; $p<0.05$ was considered to be statistically significant.

\section{Supplementary Files}

This is a list of supplementary files associated with this preprint. Click to download.

- SupplementTable7.docx

- SupplementTable7.docx

- SupplementTable7.docx

- SupplementTable6.docx

- SupplementTable6.docx

- SupplementTable6.docx

- SupplementTable5.docx

- SupplementTable5.docx

- SupplementTable5.docx

- SupplementTable4.docx

- SupplementTable4.docx

- SupplementTable4.docx

- SupplementTable3.docx

- SupplementTable3.docx

- SupplementTable3.docx

- SupplementTable2.docx

- SupplementTable2.docx

- SupplementTable2.docx

- SupplementTable1.docx

- SupplementTable1.docx

- SupplementTable1.docx 